\title{
THE KOSZUL DUAL OF THE RING OF THREE COMMUTING MATRICES
}

\author{
FREYJA HREINSDÓTTIR ${ }^{*}$
}

\begin{abstract}
Let $X=\left(x_{i j}\right), \quad Y=\left(y_{i j}\right)$ and $Z=\left(z_{i j}\right)$ be generic $n$ by $n$ matrices. Let $k$ be a field with char $k \neq 2,3, S=k\left[x_{11}, \ldots, x_{n n}, y_{11}, \ldots, y_{n n}, z_{11}, \ldots, z_{n n}\right]$ and let $I$ be the ideal generated by the entries of the matrices $X Y-Y X, X Z-Z X$ and $Y Z-Z Y$. We study the Koszul dual of the ring $R=S / I$ and show that for $n \geq 3$ this is the enveloping algebra of a nilpotent Lie algebra. We also give the dimension of the Lie algebra in each degree.
\end{abstract}

\section{Introduction}

The Koszul dual of a ring $R$ is the algebra generated by $\operatorname{Ext}_{R}^{1}(k, k)$ (as a subalgebra of the Yoneda algebra $\operatorname{Ext}_{R}^{*}(k, k)$ ) and denoted by $R^{!}$. It is known that $R^{!}=U(\mathfrak{g})$, the enveloping algebra of a graded Lie algebra $\mathfrak{g}$ (see [1] for references). In [3] a description on how to calculate $R^{!}$is given. For the ring defined in the abstract we get

$$
R^{!}=\frac{k\left\langle X_{i j}, Y_{i j}, Z_{i j}\right\rangle}{\operatorname{Rel}}
$$

i.e. $R^{!}$is a quotient of the free associative algebra on $X_{i j}, Y_{i j}, Z_{i j}$ which are variables dual to $x_{i j}, y_{i j}, z_{i j}$. The ideal Rel is generated by linear combinations of the graded commutators $[U, V]$ and $U^{2}$, where $U, V \in\left\{X_{i j}, Y_{i j}, Z_{i j}\right\}$. The graded commutator is defined as

$$
[a, b]=a b-(-1)^{|a||b|} b a
$$

We compute the generators of Rel by solving a certain system of equations. Details on how to do this are given in [1].

The graded Lie algebra $\mathfrak{g}$ is the quotient of the free graded Lie algebra on $X_{i j}, Y_{i j}, Z_{i j}$ divided by the ideal of relations Rel. Write $\mathfrak{g}=\mathfrak{g}_{1} \oplus \mathfrak{g}_{2} \oplus \cdots$. We have $\operatorname{dim} \mathfrak{g}_{1}=3 n^{2}=$ the number of variables. We calculate a generating set for the Lie algebra, one degree at a time and show the following: 
THEOREM 1. If $n \geq 3$ then the Lie algebra $\mathfrak{g}$ is nilpotent of index 4 and $\operatorname{dim} \mathfrak{g}_{1}=3 n^{2}, \operatorname{dim} \mathfrak{g}_{2}=3\left(n^{2}-1\right), \operatorname{dim} \mathfrak{g}_{3}=n^{2}+7$ and $\operatorname{dim} \mathfrak{g}_{4}=3$.

In our calculations we frequently use the graded Jacobi identity

$$
(-1)^{|a||c|}[a,[b, c]]+(-1)^{|b||a|}[b,[c, a]]+(-1)^{|c||b|}[c,[a, b]]=0
$$

In the next 4 sections we calculate a generating set for the Lie algebra. In section 7 we summarize the multiplication and in section 8 we show that the generating set is a basis. For the convenience of the reader we give the details of the multiplication for the case $n=3$ in Appendix 2.

\section{Relations in the dual ring and Lie monomials of degree 2}

In [1] we give the relations for two commuting matrices. There the ideal is generated by the entries of one matrix $X Y-Y X$. Now we have 3 sets of the same type of generators in which the monomials do not mix. We therefore get 3 sets of relations similar to the ones in [1].

For the sake of claritiy we introduce a new multigrading in the dual ring defined in the following way: a Lie monomial $m$ has the degree $\left(n_{1}, n_{2}, n_{3}\right)$ if $n_{1}=X$-degree of $m, n_{2}=Y$-degree of $m$ and $n_{3}=Z$-degree of $m$.

Monomials of degree 2 that do not occur in the generators of $I$ give zero Lie monomials in the dual ring so we clearly have the following relations:

$$
\begin{aligned}
& {\left[X_{i j}, X_{r s}\right]=0} \\
& {\left[Y_{i j}, Y_{r s}\right]=0} \\
& {\left[Z_{i j}, Z_{r s}\right]=0 .}
\end{aligned}
$$

So all Lie monomials of degrees $(2,0,0),(0,2,0)$ and $(0,0,2)$ are 0 in the dual ring. We also get

$$
\begin{array}{ll}
{\left[X_{i j}, Y_{r s}\right]=0} & \text { if } \mathrm{j} \neq r \text { and } i \neq s \\
{\left[X_{i j}, Y_{i j}\right]=0} & \text { for all }(i, j)
\end{array}
$$

and similarly for Lie monomials of degrees $(1,0,1)$ and $(0,1,1)$. The generators from $X Y-Y X$ give rise to the following relations:

$$
\begin{aligned}
& {\left[X_{i r}, Y_{r j}\right]-\left[X_{i s}, Y_{s j}\right]=0 \quad r, s, i, j \in\{1, \ldots, n\}, i \neq j} \\
& {\left[X_{i r}, Y_{r j}\right]+\left[X_{r j}, Y_{i r}\right]=0 \quad r, i, j \in\{1, \ldots, n\}, i \neq j} \\
& {\left[X_{i j}, Y_{j i}\right]+\left[X_{j i}, Y_{i j}\right]=0 \quad i, j \in\{1, \ldots, n\}} \\
& {\left[X_{1 i}, Y_{i 1}\right]-\left[X_{1 j}, Y_{j 1}\right]+\left[X_{i j}, Y_{j i}\right]=0 \quad i, j \in\{2, \ldots, n\} .}
\end{aligned}
$$

We see that the only Lie monomials of degree $(1,1,0)$ that possibly are nonzero in the dual ring may be written on the form $\left[X_{i r}, Y_{r j}\right],\left[X_{s j}, Y_{i s}\right]$ and 
$\left[X_{i j}, Y_{j i}\right]$ with $i \neq j$. Relation (1) gives that $\left[X_{i r}, Y_{r j}\right]$ is independent of $r$ so we can define

$$
a_{i j}:=\left[X_{i r}, Y_{r j}\right] \quad \text { for } i \neq j .
$$

Relations (1) and (2) give that $a_{i j}$ can also be written as $-\left[X_{s j}, Y_{i s}\right]$ for any $s$. Relations (3) and (4) give that any Lie monomial of the form $\left[X_{i j}, Y_{j i}\right]$ with $i \neq 1$ and $j \neq 1$, can be written in terms of Lie monomials of the form $\left[X_{1 j}, Y_{j 1}\right]$ and $\left[X_{1 i}, Y_{i 1}\right]$ so we define

$$
\alpha_{i}:=\left[X_{1 i}, Y_{i 1}\right] \quad \text { for } i=2, \ldots, n .
$$

We now have a generating set for degree $(1,1,0)$ with $n^{2}-1$ elements. This is a basis for the Lie monomials of degree $(1,1,0)$ (see below).

The generators from $X Z-Z X$ and $Y Z-Z Y$ give relations analogous to (1)-(4) and as in the case of Lie monomials of degree $(1,1,0)$ we define for Lie monomials of degree $(1,0,1)$

$$
\begin{aligned}
b_{i j} & :=\left[X_{i r}, Z_{r j}\right] \quad \text { for any } i \neq j \\
\beta_{i} & :=\left[X_{1 i}, Z_{i 1}\right] \text { for } i=2, \ldots, n
\end{aligned}
$$

and for Lie monomials of degree $(0,1,1)$

$$
\begin{aligned}
d_{i j} & :=\left[Y_{i r}, Z_{r j}\right] \quad \text { for any } i \neq j \\
\delta_{i} & :=\left[Y_{1 i}, Z_{i 1}\right] \quad \text { for } \mathrm{i}=2, \ldots, n .
\end{aligned}
$$

We now have that the $3\left(n^{2}-1\right)$ Lie monomials

$$
\begin{array}{rl}
a_{i j}, b_{i j}, d_{i j} & i, j \in\{1, \ldots, n\}, i \neq j \\
\alpha_{i}, \beta_{i}, \delta_{i} & i \in\{2, \ldots, n\}
\end{array}
$$

generate degree 2. In [3] we have a theorem (2.5) which gives that dim $\mathfrak{g}_{2}$ is equal to the number of generators in a minimal generating set for the ideal $I$. By looking at the monomials occurring in the generators of $I$ it is easy to see that $I$ is minimally generated by $3\left(n^{2}-1\right)$ elements (for the details of this argument see [1]). So our generating set is a basis for degree 2 .

\section{Degree 3}

As the Lie algebra is generated by its elements of degree 1 the Lie monomials in degree 3 consist of products of Lie monomials of degree 2 and the variables. We divide degree 3 into 2 cases. In the first case (subsection (3.1)) we consider Lie monomials of multidegrees $(2,1,0),(1,2,0),(2,0,1),(1,0,2)$, $(0,2,1)$ and $(0,1,2)$. It turns out that there is one Lie monomial of each of these multidegrees so this case gives 6 Lie monomials of degree 3. In the second case (subsection (3.2)) we consider multidegree $(1,1,1)$. Our compu- 
tations will show that this divides naturally into 2 parts, one giving at most $n^{2}-n$ monomials of degree 3 and the other giving at most $n+1$ monomials of degree 3 .

So the total number of Lie monomials in degree 3 is at most $n^{2}+7$.

\subsection{Mixed multidegrees}

In [1] we had two commuting matrices $X$ and $Y$. There we showed that:

$$
\begin{array}{ll}
{\left[X_{r s}, a_{i j}\right] \neq 0} & \text { iff } r=j \text { and } s=i \\
{\left[X_{r s}, \alpha_{i}\right] \neq 0} & \text { iff } r=s=1 \text { or } r=s=i
\end{array}
$$

We also showed that all the nonzero ones are equal up to a sign so degree $(2,1)$ has a basis consisting of 1 element.

Here we get similarly that there is only one (up to a sign) Lie monomial of degree $(2,1,0)$, call it $\Upsilon_{X}$. We also get only one Lie monomial of degree $(1,2,0)$ call it $\Upsilon_{Y}$. By an argument in [1] we have that $\Upsilon_{X}$ and $\Upsilon_{Y}$ are nonzero.

In the same way we get that the pair $X, Z$ gives two nonzero elements, $\Xi_{X}$ of degree $(2,0,1)$ and $\Xi_{Z}$ of degree $(1,0,2)$. The pair $Y, Z$ gives $\Lambda_{Y}$ of degree $(0,2,1)$ and $\Lambda_{Z}$ of degree $(0,1,2)$.

\subsection{Multidegree $(1,1,1)$}

In this subsection we consider monomials of multidegree $(1,1,1)$. These arise as products of $a_{p s}$ and $\alpha_{s}$ by the $Z_{i j}$-variables, $b_{p s}$ and $\beta_{s}$ by the $Y_{i j^{-}}$ variables and $d_{p s}$ and $\delta_{s}$ by the $X_{i j}$-variables. In the next 2 claims we examine which of these products are 0 .

Claim 1. For $p \neq s$ we get

$$
\left[Z_{i j}, a_{p s}\right]=0 \quad \text { if } i \neq s \text { and } j \neq p
$$

Proof. We have $\left[Z_{i j}, a_{p s}\right]=\left[Z_{i j},\left[X_{p r}, Y_{r s}\right]\right]$ where $r$ can take any value. Using the Jacobi identity we get:

$$
\left[Z_{i j},\left[X_{p r}, Y_{r s}\right]\right]=-\left[X_{p r},\left[Y_{r s}, Z_{i j}\right]\right]-\left[Y_{r s},\left[Z_{i j}, X_{p r}\right]\right] .
$$

By the relations we have $\left[Y_{r s}, Z_{i j}\right]=0$ if $i \neq s$ and $j \neq r$. Since $r$ can take any value we have to have $i=s$ for it to be nonzero. Now look at the latter term, $\left[Z_{i j}, X_{p r}\right]=0$ if $i \neq r$ and $j \neq p$ and since $r$ can take any value we have to have $j=p$. So for a given pair $(i, j)$ we pick $r \neq i, r \neq j$, then we get $\left[Z_{i j}, a_{p s}\right]=0$ if $i \neq s$ and $j \neq p$.

The same argument gives

$$
\begin{array}{ll}
{\left[Y_{i j}, b_{p s}\right]=0} & \text { if } i \neq s \text { and } j \neq p \\
{\left[X_{i j}, d_{p s}\right]=0} & \text { if } i \neq s \text { and } j \neq p
\end{array}
$$


Claim 2. For $s \neq 1$ we get

$$
\left[Z_{i j}, \alpha_{s}\right]=0 \quad \text { if } i \neq 1 \text { and } j \neq 1 \text { and } i \neq s \text { and } j \neq s .
$$

Proof. We have $\left[Z_{i j}, \alpha_{s}\right]=\left[Z_{i j},\left[X_{1 s}, Y_{s 1}\right]\right]$ and using the Jacobi identity we get

$$
\left[Z_{i j},\left[X_{1 s}, Y_{s 1}\right]\right]=-\left[X_{1 s},\left[Y_{s 1}, Z_{i j}\right]\right]-\left[Y_{s 1},\left[Z_{i j}, X_{1 s}\right]\right] .
$$

The first term is 0 if $i \neq 1$ and $j \neq s$ and the second term is 0 if $i \neq s$ and $j \neq 1$.

We get a similar result for monomials of the form $\left[X_{i j}, \delta_{s}\right]$ and $\left[Y_{i j}, \beta_{s}\right]$.

The calculations that follow will show that multidegree $(1,1,1)$ divides into 2 parts. Part 1 consists of

$$
\begin{aligned}
& {\left[Z_{s j}, a_{p s}\right] \quad \text { with } j \neq p, \text { i.e. only the outer indices are equal }} \\
& {\left[Z_{i p}, a_{p s}\right] \text { with } i \neq s, \text { i.e. only the inner indices are equal }} \\
& {\left[Z_{i j}, \alpha_{s}\right] \text { with } i \neq j \text { and } i \text { or } j \text { equal to } 1 \text { or } s}
\end{aligned}
$$

and similar products for $\left[X_{i j}, d_{p s}\right],\left[Y_{i j}, b_{p s}\right],\left[X_{i j}, \delta_{s}\right]$ and $\left[Y_{i j}, \beta_{s}\right]$. We will show that this part of degree $(1,1,1)$ is generated by $n^{2}-n$ elements.

Part 2 consists of

$$
\begin{aligned}
& {\left[Z_{s p}, a_{p s}\right] \text { i.e. both the inner and the outer indices are equal }} \\
& {\left[Z_{11}, \alpha_{s}\right]} \\
& {\left[Z_{s s}, \alpha_{s}\right]}
\end{aligned}
$$

and similar products for $\left[X_{i j}, d_{p s}\right],\left[Y_{i j}, b_{p s}\right],\left[X_{i j}, \delta_{s}\right]$ and $\left[Y_{i j}, \beta_{s}\right]$. We will show that this part of degree $(1,1,1)$ is generated by $n+1$ elements.

3.2.1. Part 1. In the next 3 claims we assume that $p \neq s$ (otherwise $a_{p s}$ is not defined).

Claim 3. For $i \neq s$ we have:

$$
\left[Z_{i p}, a_{p s}\right]=\left[Z_{i l}, a_{l s}\right] \quad \text { for any } p \neq s \text { and } l \neq s
$$

Proof. Pick $j \neq i, j \neq s$ and write $a_{p s}=\left[X_{p j}, Y_{j s}\right]$. Using the Jacobi identity we get the following

$$
\left[Z_{i p},\left[X_{p j}, Y_{j s}\right]\right]=-\left[X_{p j},\left[Y_{j s}, Z_{i p}\right]\right]-\left[Y_{j s},\left[Z_{i p}, X_{p j}\right]\right]
$$

Since $i \neq s$ we have that $\left[Y_{j s}, Z_{i p}\right] \neq 0$ if and only if $j=p$ and in that case we get that the first term is $-\left[X_{j j},\left[Y_{j s}, Z_{i j}\right]\right]=\left[X_{j j}, d_{i s}\right]$. By claim 1 we have that since $j \neq s$ and $j \neq i,\left[X_{j j}, d_{i s}\right]=0$. So the first term is always 0 i.e. $\left[Z_{i p}, a_{p s}\right]=-\left[Y_{j s},\left[Z_{i p}, X_{p j}\right]\right]$. By the relations we have $\left[Z_{i p}, X_{p j}\right]=\left[Z_{i l}, X_{l j}\right]$ for any $l$. The Jacobi identity gives 


$$
-\left[Y_{j s},\left[Z_{i l}, X_{l j}\right]\right]=\left[X_{l j},\left[Y_{j s}, Z_{i l}\right]\right]+\left[Z_{i l},\left[X_{l j}, Y_{j s}\right]\right]
$$

and $\left[X_{l j},\left[Y_{j s}, Z_{i l}\right]\right]=0$ if $j \neq l$. If $j=l$ we get $-\left[X_{j j}, d_{i s}\right]=0$. Hence we have shown $\left[Z_{i p}, a_{p s}\right]=\left[Z_{i l},\left[X_{l j}, Y_{j s}\right]\right]$. If $l \neq s$ then this is equal to $\left[Z_{i l}, a_{l s}\right]$.

Similarly we get for $i \neq s,\left[Z_{p i}, a_{s p}\right]=\left[Z_{l i}, a_{s l}\right]$ for any $l \neq s, p \neq s$. We now prove

Claim 4. Let $p \neq s, p \neq i$ and $i \neq s$. Then we have

$$
\left[Z_{i p}, a_{p s}\right]=-\left[Z_{p s}, a_{i p}\right]
$$

Proof. We have by claim 3 that $\left[Z_{i p}, a_{p s}\right]=\left[Z_{i i}, a_{i s}\right]$. We can write $a_{i s}=\left[X_{i i}, Y_{i s}\right]$ so using the Jacobi identity we get

$$
\left[Z_{i i}, a_{i s}\right]=\left[Z_{i i},\left[X_{i i}, Y_{i s}\right]\right]=-\left[Y_{i s},\left[Z_{i i}, X_{i i}\right]\right]-\left[X_{i i},\left[Y_{i s}, Z_{i i}\right]\right] .
$$

The first term is 0 and $-\left[X_{i i},\left[Y_{i s}, Z_{i i}\right]\right]=\left[X_{i i}, d_{i s}\right]$. We can write $d_{i s}=\left[Y_{i p}, Z_{p s}\right]$ so using the Jacobi identity we get

$$
\left[X_{i i}, d_{i s}\right]=\left[X_{i i},\left[Y_{i p}, Z_{p s}\right]\right]=-\left[Y_{i p},\left[Z_{p s}, X_{i i}\right]\right]-\left[Z_{p s},\left[X_{i i}, Y_{i p}\right]\right] .
$$

Since $i \neq s$ and $p \neq i,\left[Z_{p s}, X_{i i}\right]=0$ so the first term is 0 . The last term is $-\left[Z_{p s},\left[X_{i i}, Y_{i p}\right]\right]=-\left[Z_{p s}, a_{i p}\right]$ so we have proved the claim.

Using the same argument we get the same results as in claim 3 and claim 4 for elements of the form $\left[Y_{i j}, b_{p s}\right]$ and $\left[X_{i j}, d_{p s}\right]$ and we can prove the following

Claim 5. For any $i \neq s$ and $p \neq s$ we have

$$
\left[Z_{i p}, a_{p s}\right]=\left[X_{i p}, d_{p s}\right]=-\left[Y_{i p}, b_{p s}\right]
$$

and for $i \neq p$ this is equal to

$$
-\left[Z_{p s}, a_{i p}\right]=-\left[X_{p s}, d_{i p}\right]=\left[Y_{p s}, b_{i p}\right]
$$

Proof. In the proof of claim 4 we got $\left[Z_{i p}, a_{p s}\right]=\left[X_{i i}, d_{i s}\right]$. By claim 3 we have $\left[X_{i i}, d_{i s}\right]=\left[X_{i p}, d_{p s}\right]$ so $\left[Z_{i p}, a_{p s}\right]=\left[X_{i p}, d_{p s}\right]$. Write $d_{i s}=\left[Y_{i i}, Z_{i s}\right]$ then

$$
\left[X_{i i}, d_{i s}\right]=\left[X_{i i},\left[Y_{i i}, Z_{i s}\right]\right] \stackrel{\mathrm{Jac}}{=}-\left[Y_{i i},\left[Z_{i s}, X_{i i}\right]\right]-\left[Z_{i s},\left[X_{i i}, Y_{i i}\right]\right] .
$$

The last term is 0 and $-\left[Y_{i i},\left[Z_{i s}, X_{i i}\right]\right]=-\left[Y_{i i}, b_{i s}\right]=-\left[Y_{i p}, b_{p s}\right]$ by claim 3. So we have shown

$$
\left[Z_{i p}, a_{p s}\right]=\left[X_{i p}, d_{p s}\right]=-\left[Y_{i p}, b_{p s}\right] .
$$

The rest of this claim now follows from claim 4 .

REMARK. What we have proved is that for $p \neq s, i \neq s$ we have 


$$
\left[X_{i p},\left[Y_{p r}, Z_{r s}\right]\right]=\left[Z_{i p},\left[X_{p r}, Y_{r s}\right]\right]=-\left[Y_{i p},\left[Z_{p r}, X_{r s}\right]\right]
$$

and for $i \neq p$ this is equal to

$$
-\left[X_{p s},\left[Y_{i r}, Z_{r p}\right]\right]=-\left[Z_{p s},\left[X_{i r}, Y_{r p}\right]\right]=\left[Y_{p s},\left[Z_{i r}, X_{r p}\right]\right]
$$

We can now define

$$
\Gamma_{i s}=\left[Z_{i p}, a_{p s}\right] \quad \text { for any } i \neq s \text { and any } p .
$$

The total number of elements in degree 3 that are of this form is $n^{2}-n$.

Now we consider elements of the form $\left[Z_{i j}, \alpha_{s}\right]$ with $i \neq j$. We have from claim (2) that this is 0 if $i \neq 1$ and $i \neq s$ and $j \neq 1$ and $j \neq s$.

If $i=1, j \neq 1$ and $j \neq s$ we get:

$$
\left[Z_{1 j}, \alpha_{s}\right]=-\left[X_{1 s},\left[Y_{s 1}, Z_{1 j}\right]\right]=-\left[X_{1 s}, d_{s j}\right]=-\Gamma_{1 j} .
$$

If $i=1$ and $j=s$ we write (this is possible because of relation (4))

$$
\alpha_{s}=\left[X_{1 s}, Y_{s 1}\right]=\left[X_{1 r}, Y_{r 1}\right]-\left[X_{s r}, Y_{r s}\right] \quad \text { for some } r \neq s
$$

then

$$
\begin{aligned}
{\left[Z_{1 s}, \alpha_{s}\right]=} & {\left[Z_{1 s},\left[X_{1 r}, Y_{r 1}\right]\right]-\left[Z_{1 s},\left[X_{s r}, Y_{r s}\right]\right] } \\
\stackrel{\text { Jac }}{=} & -\left[X_{1 r},\left[Y_{r 1}, Z_{1 s}\right]\right]-\left[Y_{r 1},\left[Z_{1 s}, X_{1 r}\right]\right] \\
& +\left[X_{s r},\left[Y_{r s}, Z_{1 s}\right]\right]+\left[Y_{r s},\left[Z_{1 s}, X_{s r}\right]\right] \\
= & -\left[X_{1 r}, d_{r s}\right]-\left[Y_{r s}, b_{1 r}\right] \\
= & -\Gamma_{1 s}-\Gamma_{1 s}=-2 \Gamma_{1 s}
\end{aligned}
$$

The rest of the cases are calculated similarly. We get:

$$
\left[Z_{i j}, \alpha_{s}\right]=\left\{\begin{aligned}
& 0 \text { if } i \neq 1 \text { and } i \neq s \text { and } j \neq 1 \text { and } j \neq s \\
&-\Gamma_{1 j} \text { if } i=1 \text { and } j \neq 1 \text { and } j \neq s \\
& \Gamma_{s j} \text { if } i=s \text { and } j \neq 1 \text { and } j \neq s \\
& \Gamma_{i 1} \text { if } j=1 \text { and } i \neq 1 \text { and } i \neq s \\
&-\Gamma_{i s} \text { if } j=s \text { and } i \neq 1 \text { and } i \neq s \\
&-2 \Gamma_{1 s} \text { if } i=1 \text { and } j=s \\
& 2 \Gamma_{s 1} \text { if } i=s \text { and } j=1
\end{aligned}\right.
$$

We get similar results for elements of the form $\left[Y_{i j}, \beta_{s}\right]$ and $\left[X_{i j}, \delta_{s}\right]$.

3.2.2. Part 2. What remains to be considered are the elements of the form $\left[Z_{11}, \alpha_{s}\right],\left[Z_{s s}, \alpha_{s}\right],\left[X_{11}, \delta_{s}\right],\left[X_{s s}, \delta_{s}\right],\left[Y_{11}, \beta_{s}\right],\left[Y_{s s}, \beta_{s}\right]$ and $\left[Z_{i j}, a_{j i}\right],\left[X_{i j}, d_{j i}\right]$, $\left[Y_{i j}, b_{j i}\right]$. We are going to prove a number of claims on how these elements relate to each other and show that we need a generating set consisting of $n+1$ elements to generate all of them. 
Claim 6. The elements $\left[Z_{r r},\left[X_{r s}, Y_{s r}\right]\right]$, with $r, s \in\{1, \ldots, n\}, r \neq s$, are all equal.

Proof. We have

$$
\begin{aligned}
{\left[Z_{11}, \alpha_{s}\right] } & =\left[Z_{11},\left[X_{1 s}, Y_{s 1}\right]\right] \stackrel{\mathrm{Jac}}{=}-\left[X_{1 s},\left[Y_{s 1}, Z_{11}\right]\right]-\left[Y_{s 1},\left[Z_{11}, X_{1 s}\right]\right] \\
& =-\left[X_{1 s}, d_{s 1}\right]+\left[Y_{s 1}, b_{1 s}\right]
\end{aligned}
$$

and similarly

$$
\left[Z_{s s}, \alpha_{s}\right]=\left[Z_{s s},\left[X_{1 s}, Y_{s 1}\right]\right]=\left[X_{1 s}, d_{s 1}\right]-\left[Y_{s 1}, b_{1 s}\right]
$$

so $\left[Z_{11}, \alpha_{s}\right]=-\left[Z_{s s}, \alpha_{s}\right]$.

By relation (4) we have $\alpha_{r}-\alpha_{s}+\left[X_{r s}, Y_{s r}\right]=0$ for any $r \neq s, r \neq 1$. We multiply this equation by $Z_{11}$ and get

$$
\left[Z_{11}, \alpha_{r}\right]-\left[Z_{11}, \alpha_{s}\right]+\left[Z_{11},\left[X_{r s}, Y_{s r}\right]\right]=0 .
$$

By using the Jacobi identity we get $\left[Z_{11},\left[X_{r s}, Y_{s r}\right]\right]=0$ so this gives $\left[Z_{11}, \alpha_{r}\right]$ $=\left[Z_{11}, \alpha_{s}\right]$. So we have shown

$$
-\left[Z_{s s}, \alpha_{s}\right]=\left[Z_{11}, \alpha_{s}\right]=\left[Z_{11}, \alpha_{r}\right]=-\left[Z_{r r}, \alpha_{r}\right] \text { for any } r, s \neq 1 .
$$

Now look at $\left[Z_{r r},\left[X_{r s}, Y_{s r}\right]\right]$ with $r \neq s, r \neq 1$ and $s \neq 1$. We have $\alpha_{r}-\alpha_{s}+\left[X_{r s}, Y_{s r}\right]=0$. Multiply through this equation by $Z_{r r}$. Since $\left[Z_{r r}, \alpha_{s}\right]=0$ this gives

$$
-\left[Z_{r r}, \alpha_{r}\right]=\left[Z_{r r},\left[X_{r s}, Y_{s r}\right]\right] .
$$

So we have proved the claim.

Similarly we get that there is only one element of the type $\left[X_{r r},\left[Y_{r s}, Z_{s r}\right]\right]$ and one of the type $\left[Y_{r r},\left[X_{r s}, Z_{s r}\right]\right]$. We define

$$
\begin{aligned}
H & =\left[Z_{r r},\left[X_{r s}, Y_{s r}\right]\right] & & \text { for any } r \neq s \\
F & =\left[X_{r r},\left[Y_{r s}, Z_{s r}\right]\right] & & \text { for any } r \neq s \\
E & =\left[Y_{r r},\left[X_{r s}, Z_{s r}\right]\right] & & \text { for any } r \neq s
\end{aligned}
$$

and for any $i \neq j$

$$
\begin{aligned}
H_{i j} & =\left[Z_{i j}, a_{j i}\right] \\
F_{i j} & =\left[X_{i j}, d_{j i}\right] \\
E_{i j} & =\left[Y_{i j}, b_{j i}\right]
\end{aligned}
$$

We have $3\left(n^{2}-n\right)+3$ elements. We are going to show that these can all be written in terms of $H, F$ and $H_{12}, \ldots, H_{1 n}$. 
By using the Jacobi identity we easily get for any $r \neq s$

$$
\begin{aligned}
H & =-F_{s r}+E_{r s} \\
F & =E_{r s}-H_{s r} \\
E & =H_{s r}+F_{r s}
\end{aligned}
$$

So $E-F=H_{r s}+F_{s r}-E_{r s}+H_{s r}=H_{r s}+H_{s r}-H$. By using the Jacobi identity we get

$$
H_{i j}=-F_{j r}+E_{r i} .
$$

From the above we have $F_{j r}=E_{r j}-H$ and $E_{r i}=F_{i r}+H$ then $H_{i j}=-\left(E_{r j}-H\right)+\left(F_{i r}+H\right)=-H_{j i}+2 H$ so

$$
H_{i j}+H_{j i}=2 H
$$

and

$$
E-F=H \text {. }
$$

We can now prove

Claim. 7. For any $i \neq 1, j \neq 1$ and $i \neq j$ we have

$$
H_{1 i}-H_{1 j}+H_{i j}=H
$$

Proof. We have

$$
\begin{aligned}
H_{1 i} & =-F_{i r}+E_{r 1} \\
H_{1 j} & =-F_{j r}+E_{r 1} \\
H_{i j} & =-F_{j r}+E_{r i}
\end{aligned}
$$

so $H_{1 i}-H_{1 j}+H_{i j}=-F_{i r}+E_{r i}=H$.

We can now write this part of degree 3 in terms of $H, H_{12}, \ldots, H_{1 n}$ and $F$ :

$$
\begin{aligned}
H_{i j} & =H-H_{1 i}+H_{1 j} & & \text { for any } i, j \in\{2, \ldots, n\}, i \neq j \\
H_{i 1} & =2 H-H_{1 i} & & \text { for any } i \in\{2, \ldots, n\} \\
E_{i j} & =F+H_{j i}=F+H-H_{1 j}+H_{1 i} & & \text { for any } i, j \in\{2, \ldots, n\}, i \neq j \\
E_{1 j} & =F+H_{j 1}=F+2 H-H_{1 j} & & \text { for any } j \in\{2, \ldots, n\} \\
E_{i 1} & =F+H_{1 i} & & \text { for any } i \in\{2, \ldots, n\} \\
F_{i j} & =E_{j i}-H=F+H_{1 j}-H_{1 i} & & \text { for any } i, j \in\{2, \ldots, n\}, i \neq j \\
F_{1 j} & =E_{j 1}-H=F+H_{1 j}-H & & \text { for any } j \in\{2, \ldots, n\} \\
F_{i 1} & =E_{1 i}-H=F+H-H_{1 i} & & \text { for any } i \in\{2, \ldots, n\} \\
E & =F+H & &
\end{aligned}
$$

This means that it suffices with $n+1$ elements to generate this part of degree 3 . So we have shown that to generate multidegree $(1,1,1)$ it suffices with 
$n^{2}-n+n+1=n^{2}+1$ generators. In subsection 3.1 we got 6 generators so the total number of generators in degree 3 is at most $n^{2}+1+6=n^{2}+7$.

\section{Degree 4}

We multiply the generators in degree 3 by the variables. We are going to show that this results in 3 Lie monomials of degrees $(2,1,1),(1,2,1)$ and $(1,1,2)$ respectively.

We first consider the generators from subsection 3.1. We know from [1] that multiplying $\Upsilon_{X}$ and $\Upsilon_{Y}$ with the $X$-or $Y$-variables gives 0 , multiplying $\Xi_{X}$ and $\Xi_{Z}$ with the $X$-or $Z$-variables gives 0 and multiplying $\Lambda_{Y}$ and $\Lambda_{Z}$ with the $Y$-or $Z$-variables gives 0 . So the product of this part of degree 3 with the variables can only result in elements of multidegrees $(1,1,2)$, $(1,2,1)$ and $(2,1,1)$.

The product of the elements of multidegree $(1,1,1)$ by the variables can also only result in elements of multidegrees $(1,1,2),(1,2,1)$ and $(2,1,1)$ so these are the only possible multidegrees that can occur in degree 4 . We study multidegree $(1,1,2)$ and show that it is generated by one element. By symmetry the same result will follow for the other multidegrees.

We first prove:

Claim 8. Let $i \neq s$ then

$$
\left[Z_{l k}, \Gamma_{i s}\right]=0 \quad \text { if } l \neq s \text { or } k \neq i .
$$

Proof. Write $\Gamma_{i s}=\left[Z_{i p}, a_{p s}\right]$ with $p \neq k$. We get

$$
\left[Z_{l k}, \Gamma_{i s}\right]=\left[Z_{l k},\left[Z_{i p}, a_{p s}\right]\right]=\left[Z_{i p},\left[a_{p s}, Z_{l k}\right]\right]-\left[a_{p s},\left[Z_{l k}, Z_{i p}\right]\right]
$$

The last term is 0 and by claim 1 the first term is 0 if $l \neq s$ (since $p \neq k$ ). By claim 4 we can also write $\Gamma_{i s}=-\left[Z_{r s}, a_{i r}\right]$ with $r \neq l$. We get

$$
\left[Z_{l k}, \Gamma_{i s}\right]=-\left[Z_{r s},\left[a_{i r}, Z_{l k}\right]\right]+\left[a_{i r},\left[Z_{r s}, Z_{l k}\right]\right]
$$

and the first term is zero if $k \neq i$. The last term is 0 . So we have shown that $\left[Z_{l k}, \Gamma_{i s}\right]=0$ if $l \neq s$ or $k \neq i$.

Later we will show that $\left[Z_{s i}, \Gamma_{i s}\right]=\left[Z_{r j}, \Gamma_{j r}\right]$ for any $(j, r)$ with $j \neq r$.

Now multiply $H$ with $Z_{k l}$. We get

Claim 9.

$$
\left[Z_{k l}, H\right]=0 \quad \text { for any }(k, l) .
$$

Proof. We can write $H=\left[Z_{r r},\left[X_{r s}, Y_{s r}\right]\right]$ for any $r \neq s$. Pick $r \neq k, r \neq l$ and $s \neq l$ then 


$$
\left[Z_{k l},\left[Z_{r r},\left[X_{r s}, Y_{s r}\right]\right]=-\left[\left[X_{r s}, Y_{s r}\right],\left[Z_{k l}, Z_{r r}\right]\right]+\left[Z_{r r},\left[\left[X_{r s}, Y_{s r}\right], Z_{k l}\right]\right]\right.
$$

The first term is 0 and the last term is 0 if $s \neq k$ (since $k \neq r$ and $l \neq r, l \neq s$ ). If $n \geq 4$ then we can pick $s \neq k$ and the result follows. For $n=3$ we may have $s=k$.

In that case we get

$$
-\left[Z_{s l},\left[X_{r s}, Y_{s r}\right]\right] \stackrel{\mathrm{Jac}}{=}\left[Y_{s r},\left[Z_{s l}, X_{r s}\right]\right]=\left[Y_{s r}, b_{r l}\right]=-\Gamma_{s l}
$$

and $\left[Z_{r r},-\Gamma_{s l}\right]=0$ since $r \neq s, r \neq l$ (by claim 8 ) so $\left[Z_{k l}, H\right]=0$ for any $(k, l)$.

We also get

Claim 10. For $i \neq j$ we have

$$
\left[Z_{k l}, H_{i j}\right]= \begin{cases}{\left[Z_{i j}, \Gamma_{j i}\right]} & \text { if } k=i=l \\ -\left[Z_{i j}, \Gamma_{j i}\right] & \text { if } k=j=l \\ 0 & \text { otherwise }\end{cases}
$$

Proof. By definition $H_{i j}=\left[Z_{i j}, a_{j i}\right]$ and the Jacobi identity gives

$$
\left[Z_{k l},\left[Z_{i j}, a_{j i}\right]\right]=\left[Z_{i j},\left[a_{j i}, Z_{k l}\right]\right]-\left[a_{j i},\left[Z_{k l}, Z_{i j}\right]\right] .
$$

The last term is 0 and $\left[a_{j i}, Z_{k l}\right]=0$ if $k \neq i$ and $l \neq j$. If $k=i$ and $l \neq j$ then we get $\left[Z_{i j},\left[a_{j i}, Z_{i l}\right]\right]=\left[Z_{i j}, \Gamma_{j l}\right]$. By claim 8 we have that this is 0 if $l \neq i$. The case $k \neq i, l=j$ is similar. If $k=i, l=j$ and $i \neq j$ we have $\left[Z_{i j}, H_{i j}\right]$. By equation (5) we can write $H_{i j}=2 H-H_{j i}$ so

$$
\begin{gathered}
{\left[Z_{i j}, H_{i j}\right]=2\left[Z_{i j}, H\right]-\left[Z_{i j}, H_{j i}\right]=-\left[Z_{i j}, H_{j i}\right]=-\left[Z_{i j},\left[Z_{j i}, a_{i j}\right]\right]} \\
\stackrel{\mathrm{Jac}}{=}-\left[Z_{j i},\left[a_{i j}, Z_{i j}\right]\right]=0 \quad \text { since } i \neq j .
\end{gathered}
$$

From this we easily get

Claim 11. For any $i \neq j$ we have

$$
\left[Z_{i j}, \Gamma_{j i}\right]=\left[Z_{j i}, \Gamma_{i j}\right]
$$

Proof. We have

$$
\left[Z_{i j}, \Gamma_{j i}\right]=\left[Z_{i i}, H_{i j}\right]=\left[Z_{i i}, 2 H-H_{j i}\right]=-\left[Z_{i i}, H_{j i}\right]=\left[Z_{j i}, \Gamma_{i j}\right]
$$

We also get

Claim 12. Let $i \neq s$. For any $p \neq s, p \neq i$ we have

$$
\left[Z_{s i}, \Gamma_{i s}\right]=\left[Z_{p s}, \Gamma_{s p}\right]
$$

Proof. By claim 4 we can write $\Gamma_{i s}=-\left[Z_{p s}, a_{i p}\right]$ then 


$$
\left[Z_{s i}, \Gamma_{i s}\right]=-\left[Z_{s i},\left[Z_{p s}, a_{i p}\right]\right] \stackrel{\mathrm{Jac}}{=}-\left[Z_{p s},\left[a_{i p}, Z_{s i}\right]\right]=\left[Z_{p s}, \Gamma_{s p}\right]
$$

The last two claims give that all the elements of the form $\left[Z_{i j}, \Gamma_{j i}\right]$ are equal so we can define

$$
\Phi_{Z}:=\left[Z_{s i}, \Gamma_{i s}\right]
$$

We now prove:

Claim 13.

$$
\left[Z_{i j}, F\right]=0 \quad \text { for any }(i, j)
$$

Proof. We can write $F=\left[X_{r r},\left[Y_{r s}, Z_{s r}\right]\right]$ for any $r$ and $s$ with $r \neq s$ so

$$
\left[Z_{i j}, F\right] \stackrel{\mathrm{Jac}}{=}-\left[\left[Y_{r s}, Z_{r s}\right],\left[Z_{i j}, X_{r r}\right]\right]+\left[X_{r r},\left[\left[Y_{r s}, Z_{s r}\right], Z_{i j}\right]\right] .
$$

By subsection 3.1 we have $\left[Z_{i j},\left[Y_{r s}, Z_{s r}\right]\right]=0$ unless $i=j=r$ or $i=j=s$. If $i=j$ we pick $r$ and $s$ different from $i$ and get that both terms are 0 (since $\left[Z_{i i}, X_{r r}\right]=0$ ). For $i \neq j$ we get that the last term is 0 and the first term is 0 if we pick $r \neq i, r \neq j$.

What remains to be considered in multidegree $(1,1,2)$ is $\left[X_{p s}, \Lambda_{Z}\right]$ and $\left[Y_{p s}, \Xi_{Z}\right]$. We get

Claim 14.

$$
\left[X_{p s}, \Lambda_{Z}\right]=0 \quad \text { for any }(p, s) .
$$

Proof. Let $(p, s)$ be given. Pick $(i, j)$ such that $i \neq j, i \neq p, i \neq s$ and $j \neq p$ and write $\Lambda_{Z}=\left[Z_{i j}, d_{j i}\right]$. Then

$$
\left[X_{p s},\left[Z_{i j}, d_{j i}\right]\right] \stackrel{\mathrm{Jac}}{=}\left[Z_{i j},\left[d_{j i}, X_{p s}\right]\right]-\left[d_{j i},\left[X_{p s}, Z_{i j}\right]\right] .
$$

The last term is 0 since $i \neq s$ and $j \neq p$. The first term is also 0 if $j \neq s$. If $j=s$ then the first term becomes

$$
\left[Z_{i j},\left[d_{j i}, X_{p j}\right]\right]=\left[Z_{i j},-\Gamma_{p i}\right]=0 \quad \text { since } j \neq p(\text { claim 8) }
$$

so $\left[X_{p s},\left[Z_{i j}, d_{j i}\right]\right]=0$.

A similar argument gives that $\left[Y_{p s}, \Xi_{Z}\right]=0$ for any $(p, s)$. So we have shown that multidegree $(1,1,2)$ is generated by 1 element.

By symmetry we get that the multidegree $(2,1,1)$ is generated by one element, call it $\Phi_{X}$ and the multidegree $(1,2,1)$ is generated by one element, call it $\Phi_{Y}$. 


\section{Degree 5}

Here we look at the products of $\Phi_{X}, \Phi_{Y}$ and $\Phi_{Z}$ and the variables and show that this results in zeros, i.e. there are no elements in degree 5.

We first look at $\left[Z_{k l}, \Phi_{Z}\right]$. For any $i \neq s$ we can write $\Phi_{Z}=\left[Z_{s i}, \Gamma_{i s}\right]$. Pick $s \neq k$. Then we get

$$
\left[Z_{k l},\left[Z_{s i}, \Gamma_{i s}\right]\right]=-\left[Z_{s i},\left[\Gamma_{i s}, Z_{k l}\right]\right]-\left[\Gamma_{i s},\left[Z_{k l}, Z_{s i}\right]\right] .
$$

The last term is 0 and $\left[\Gamma_{i s}, Z_{k l}\right]=0$ since $s \neq k$ (by claim 8 ) so the first term is 0 .

To show that $\left[X_{k l}, \Phi_{Z}\right]=0$ we pick $(i, s)$ such that $i \neq s, i \neq k, i \neq l$ and $s \neq l$. We get

$$
\left[X_{k l},\left[Z_{s i}, \Gamma_{i s}\right]\right]=\left[Z_{s i},\left[\Gamma_{i s}, X_{k l}\right]\right]+\left[\Gamma_{i s},\left[X_{k l}, Z_{s i}\right]\right] .
$$

The first term is 0 since $i \neq l$ (by claim 8 ) and $\left[X_{k l}, Z_{s i}\right]=0$ since $i \neq k$ and $s \neq l$ so the last term is 0 . Similarly we get $\left[Y_{k l}, \Phi_{Z}\right]=0$ so multiplying $\Phi_{Z}$ by the variables does not give any elements in degree 5 . We easily get the same result for $\Phi_{X}$ and $\Phi_{Y}$ so $\mathfrak{g}_{5}=0$ i.e. the Lie algebra is nilpotent of index 4 .

\section{Conclusion}

We have now shown that the Lie algebra is generated by the following elements:

$$
\begin{array}{rlr}
\operatorname{deg} 1: & X_{i j}, Y_{i j}, Z_{i j} & \text { for } i, j=1, \ldots, n \\
\operatorname{deg} 2: & a_{i j}, b_{i j}, d_{i j}, & \text { for } i, j=1, \ldots, n \text { and } i \neq j \\
& \alpha_{k}, \beta_{k}, \delta_{k} & \text { for } k=2, \ldots, n . \\
\operatorname{deg} 3: & \Gamma_{i j} & \text { for } i, j=1, \ldots, n \text { and } i \neq j \\
& F, H, H_{12}, \ldots, H_{1 n} & \\
& \Upsilon_{X}, \Upsilon_{Y}, \Xi_{X}, \Xi_{Z}, \Lambda_{Y}, \Lambda_{Z} & \\
\operatorname{deg} 4: & \Phi_{X}, \Phi_{Y}, \Phi_{Z} &
\end{array}
$$

The multiplication tables are given in the next section.

\section{Multiplication tables}

In this section we give a summary of the multiplication in each degree in the Lie algebra.

\subsection{Degree 2}

We have:

$$
\begin{array}{ll}
{\left[X_{i j}, X_{r s}\right]=0} & \text { for all } i, j, r, s \in\{1, \ldots, n\} \\
{\left[Y_{i j}, Y_{r s}\right]=0} & \text { for all } i, j, r, s \in\{1, \ldots, n\} \\
{\left[Z_{i j}, Z_{r s}\right]=0} & \text { for all } i, j, r, s \in\{1, \ldots, n\}
\end{array}
$$




$$
\begin{aligned}
& {\left[X_{r s}, Y_{i j}\right]= \begin{cases}0 & \text { if } s \neq i \text { and } r \neq j \\
a_{r j} & \text { if } s=i \text { and } r \neq j \\
-a_{i s} & \text { if } s \neq i \text { and } r=j \\
\alpha_{s}-\alpha_{r} & \text { if } r=j \neq 1 \text { and } s=i \neq 1 \text { and } r \neq s \\
\alpha_{s} & \text { if } r=j=1 \text { and } s=i \neq 1 \\
-\alpha_{r} & \text { if } r=j \neq 1 \text { and } s=i=1 \\
0 & \text { if } r=s=i=j\end{cases} } \\
& {\left[X_{r s}, Z_{i j}\right]= \begin{cases}0 & \text { if } s \neq i \text { and } r \neq j \\
b_{r j} & \text { if } s=i \text { and } r \neq j \\
-b_{i s} & \text { if } s \neq i \text { and } r=j \\
\beta_{s}-\beta_{r} & \text { if } r=j \neq 1 \text { and } s=i \neq 1 \text { and } r \neq s \\
\beta_{s} & \text { if } r=j=1 \text { and } s=i \neq 1 \\
-\beta_{r} & \text { if } r=j \neq 1 \text { and } s=i=1 \\
0 & \text { if } r=s=i=j\end{cases} } \\
& {\left[Y_{r s}, Z_{i j}\right]= \begin{cases}0 & \text { if } s \neq i \text { and } r \neq j \\
d_{r j} & \text { if } s=i \text { and } r \neq j \\
-d_{i s} & \text { if } s \neq i \text { and } r=j \\
\delta_{s}-\delta_{r} & \text { if } r=j \neq 1 \text { and } s=i \neq 1 \text { and } r \neq s \\
\delta_{s} & \text { if } r=j=1 \text { and } s=i \neq 1 \\
-\delta_{r} & \text { if } r=j \neq 1 \text { and } s=i=1 \\
0 & \text { if } r=s=i=j\end{cases} }
\end{aligned}
$$

7.2. Degree 3

Let $p \neq s$ then

$$
\begin{gathered}
{\left[X_{i j}, a_{p s}\right]= \begin{cases}\Upsilon_{X} & \text { if } i=s \text { and } j=p \\
0 & \text { otherwise }\end{cases} } \\
{\left[Y_{i j}, a_{p s}\right]= \begin{cases}\Upsilon_{Y} & \text { if } i=s \text { and } j=p \\
0 & \text { otherwise }\end{cases} } \\
{\left[Z_{i j}, a_{p s}\right]= \begin{cases}0 & \text { if } i \neq s \text { and } j \neq p \\
\Gamma_{i s} & \text { if } i \neq s \text { and } j=p \\
-\Gamma_{p j} & \text { if } i=s \text { and } j \neq p \\
H-H_{1 i}+H_{1 j} & \text { if } i=s \neq 1 \text { and } j=p \neq 1 \\
H_{1 j} & \text { if } i=s=1 \text { and } j=p \neq 1 \\
2 H-H_{1 i} & \text { if } i=s \neq 1 \text { and } j=p=1 \\
0 & \text { if } i=s=j=p\end{cases} }
\end{gathered}
$$


Let $s \neq 1$ then

$$
\left[X_{i j}, \alpha_{s}\right]=\left\{\begin{array}{cc}
\Upsilon_{X} & \text { if } i=j=1 \\
-\Upsilon_{X} & \text { if } i=j=s \\
0 & \text { otherwise }
\end{array}\right.
$$

and

$$
\left[Y_{i j}, \alpha_{s}\right]= \begin{cases}\Upsilon_{Y} & \text { if } i=j=1 \\ -\Upsilon_{Y} & \text { if } i=j=s \\ 0 & \text { otherwise }\end{cases}
$$

and

$$
\left[Z_{i j}, \alpha_{s}\right]= \begin{cases}0 & \text { if } i \neq 1 \text { and } i \neq s \text { and } j \neq 1 \text { and } j \neq s \\ -\Gamma_{1 j} & \text { if } i=1, j \neq 1, j \neq s \\ \Gamma_{s j} & \text { if } i=s, j \neq 1, j \neq s \\ \Gamma_{i 1} & \text { if } j=1, i \neq 1, i \neq s \\ -\Gamma_{i s} & \text { if } j=s, i \neq 1, i \neq s \\ -2 \Gamma_{1 s} & \text { if } i=1, j=s \\ 2 \Gamma_{s 1} & \text { if } i=s, j=1 \\ H & \text { if } i=j=1 \\ -H & \text { if } i=j=s\end{cases}
$$

Let $p \neq s$ then

$$
\begin{aligned}
& {\left[X_{i j}, b_{p s}\right]= \begin{cases}\Xi_{X} & \text { if } i=s \text { and } j=p \\
0 & \text { otherwise }\end{cases} } \\
& {\left[Z_{i j}, b_{p s}\right]= \begin{cases}\Xi_{Z} & \text { if } i=s \text { and } j=p \\
0 & \text { otherwise }\end{cases} }
\end{aligned}
$$

and

$$
\left[Y_{i j}, b_{p s}\right]= \begin{cases}0 & \text { if } i \neq s \text { and } j \neq p \\ -\Gamma_{i s} & \text { if } i \neq s \text { and } j=p \\ \Gamma_{p j} & \text { if } i=s \text { and } j \neq p \\ F+H-H_{1 j}+H_{1 i} & \text { if } i=s \neq 1 \text { and } j=p \neq 1 \\ F+2 H-H_{1 j} & \text { if } i=s=1 \text { and } j=p \neq 1 \\ F+H_{1 i} & \text { if } i=s \neq 1 \text { and } j=p=1 \\ 0 & \text { if } i=s=j=p\end{cases}
$$

Let $s \neq 1$ then

$$
\left[X_{i j}, \beta_{s}\right]= \begin{cases}\Xi_{X} & \text { if } i=j=1 \\ -\Xi_{X} & \text { if } i=j=s \\ 0 & \text { otherwise }\end{cases}
$$


and

$$
\left[Z_{i j}, \beta_{s}\right]= \begin{cases}\Xi_{Z} & \text { if } i=j=1 \\ -\Xi_{Z} & \text { if } i=j=s \\ 0 & \text { otherwise }\end{cases}
$$

and

$$
\left[Y_{i j}, \beta_{s}\right]= \begin{cases}0 & \text { if } i \neq 1 \text { and } i \neq s \text { and } j \neq 1 \text { and } j \neq s \\ \Gamma_{1 j} & \text { if } i=1, j \neq 1, j \neq s \\ -\Gamma_{s j} & \text { if } i=s, j \neq 1, j \neq s \\ -\Gamma_{i 1} & \text { if } j=1, i \neq 1, i \neq s \\ \Gamma_{i s} & \text { if } j=s, i \neq 1, i \neq s \\ 2 \Gamma_{1 s} & \text { if } i=1, j=s \\ -2 \Gamma_{s 1} & \text { if } i=s, j=1 \\ F+H & \text { if } i=j=1 \\ -F-H & \text { if } i=j=s\end{cases}
$$

Let $p \neq s$ then

$$
\begin{gathered}
{\left[Y_{i j}, d_{p s}\right]= \begin{cases}\Lambda_{Y} & \text { if } i=s \text { and } j=p \\
0 & \text { otherwise }\end{cases} } \\
{\left[Z_{i j}, d_{p s}\right]= \begin{cases}\Lambda_{Z} & \text { if } i=s \text { and } j=p \\
0 & \text { otherwise }\end{cases} } \\
{\left[X_{i j}, d_{p s}\right]= \begin{cases}0 & \text { if } i \neq s \text { and } j \neq p \\
\Gamma_{i s} & \text { if } i \neq s \text { and } j=p \\
-\Gamma_{p j} & \text { if } i=s \text { and } j \neq p \\
F+H_{1 j}-H_{1 i} & \text { if } i=s \neq 1 \text { and } j=p \neq 1 \\
F-H+H_{1 j} & \text { if } i=s=1 \text { and } j=p \neq 1 \\
F+H-H_{1 i} & \text { if } i=s \neq 1 \text { and } j=p=1 \\
0 & \text { if } i=s=j=p\end{cases} }
\end{gathered}
$$

Let $s \neq 1$ then

$$
\left[Y_{i j}, \delta_{s}\right]= \begin{cases}\Lambda_{Y} & \text { if } i=j=1 \\ -\Lambda_{Y} & \text { if } i=j=s \\ 0 & \text { otherwise }\end{cases}
$$

and

$$
\left[Z_{i j}, \delta_{s}\right]= \begin{cases}\Lambda_{Z} & \text { if } i=j=1 \\ -\Lambda_{Z} & \text { if } i=j=s \\ 0 & \text { otherwise }\end{cases}
$$

and 


$$
\left[X_{i j}, \delta_{s}\right]= \begin{cases}0 & \text { if } i \neq 1 \text { and } i \neq s \text { and } j \neq 1 \text { and } j \neq s \\ -\Gamma_{1 j} & \text { if } i=1, j \neq 1, j \neq s \\ \Gamma_{s j} & \text { if } i=s, j \neq 1, j \neq s \\ \Gamma_{i 1} & \text { if } j=1, i \neq 1, i \neq s \\ -\Gamma_{i s} & \text { if } j=s, i \neq 1, i \neq s \\ -2 \Gamma_{1 s} & \text { if } i=1, j=s \\ 2 \Gamma_{s 1} & \text { if } i=s, j=1 \\ F & \text { if } i=j=1 \\ -F & \text { if } i=j=s\end{cases}
$$

\subsection{Degree 4}

Multiplying the elements

$$
\Upsilon_{X}, \Upsilon_{Y}, \Xi_{X}, \Xi_{Z}, \Lambda_{Y}, \Lambda_{Z}, F, H
$$

by the variables gives only zeros.

$$
\begin{aligned}
& {\left[X_{r s}, \Gamma_{i j}\right]= \begin{cases}\Phi_{X} & \text { if } r=j \text { and } s=i \\
0 & \text { otherwise }\end{cases} } \\
& {\left[Y_{r s}, \Gamma_{i j}\right]= \begin{cases}\Phi_{Y} & \text { if } r=j \text { and } s=i \\
0 & \text { otherwise }\end{cases} } \\
& {\left[Z_{r s}, \Gamma_{i j}\right]= \begin{cases}\Phi_{Z} & \text { if } r=j \text { and } s=i \\
0 & \text { otherwise }\end{cases} }
\end{aligned}
$$

Let $k \in\{2, \ldots, n\}$ then

$$
\left[X_{r s}, H_{1 k}\right]= \begin{cases}\Phi_{X} & \text { if } r=s=1 \\ -\Phi_{X} & \text { if } r=s=k \\ 0 & \text { otherwise }\end{cases}
$$

and

$$
\begin{aligned}
& {\left[Y_{r s}, H_{1 k}\right]= \begin{cases}\Phi_{Y} & \text { if } r=s=1 \\
-\Phi_{Y} & \text { if } r=s=k \\
0 & \text { otherwise }\end{cases} } \\
& {\left[Z_{r s}, H_{1 k}\right]= \begin{cases}\Phi_{Z} & \text { if } r=s=1 \\
-\Phi_{Z} & \text { if } r=s=k \\
0 & \text { otherwise }\end{cases} }
\end{aligned}
$$




\section{A basis for the Lie algebra}

We have shown that $\operatorname{dim} \mathfrak{g}_{1}=3 n^{2}, \operatorname{dim} \mathfrak{g}_{2}=3\left(n^{2}-1\right)$, $\operatorname{dim} \mathfrak{g}_{3} \leq n^{2}+7$ and $\operatorname{dim} \mathfrak{g}_{4} \leq 3$. In this section we show that we have equalities in degrees 3 and 4. To do that we use a method given in [2]. We construct a module that has symbols corresponding to the Lie algebra generators as a $k$-basis and then we get a 1-1 map from it to the Lie algebra.

\subsection{The module}

We let $M$ be a graded vectorspace on the following symbols:

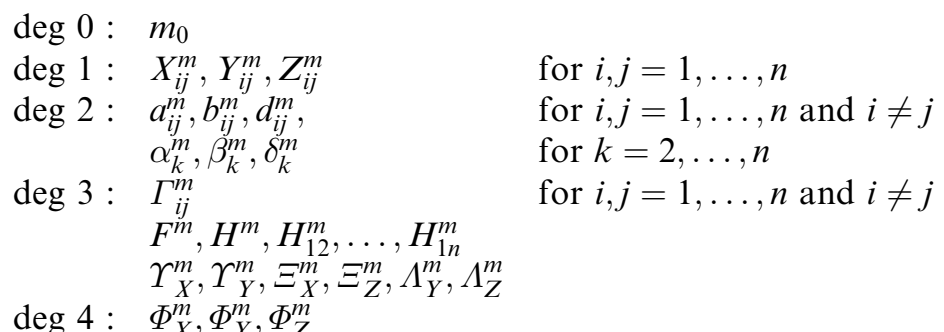

We assign a multigrading to the generators of $M$ such that multidegree $w^{m}=$ multidegree $w$ e.g. multidegree $a_{i j}^{m}=$ multidegree $a_{i j}=(1,1,0)$. Let $\mathfrak{g}_{f}$ be the free Lie algebra on $X_{i j}, Y_{i j}, Z_{i j}$. We make $M$ into a $\mathfrak{g}_{f}$-module by defining the operation of $X_{i j}, Y_{i j}$ and $Z_{i j}$ on $M$. We define

$$
\begin{aligned}
X_{i j} \cdot m_{0}:=X_{i j}^{m} & \text { for } i, j=1, \ldots, n \\
Y_{i j} \cdot m_{0}:=Y_{i j}^{m} & \text { for } i, j=1, \ldots, n \\
Z_{i j} \cdot m_{0}:=Z_{i j}^{m} & \text { for } i, j=1, \ldots, n .
\end{aligned}
$$

For the operation of the variables on the rest of the elements of $M$ we define it as the Lie algebra multiplication in $\mathfrak{g}$ with $[U$,$] replaced by U$. for $U \in\left\{X_{i j}, Y_{i j}, Z_{i j}\right\}$. That is we define e.g.

$$
X_{r s} . Y_{i j}^{m}:= \begin{cases}0 & \text { if } s \neq i \text { and } r \neq j \\ a_{r j}^{m} & \text { if } s=i \text { and } r \neq j \\ -a_{i s}^{m} & \text { if } s \neq i \text { and } r=j \\ \alpha_{s}^{m}-\alpha_{r}^{m} & \text { if } r=j \neq 1 \text { and } s=i \neq 1 \text { and } r \neq s \\ \alpha_{s}^{m} & \text { if } r=j=1 \text { and } s=i \neq 1 \\ -\alpha_{r}^{m} & \text { if } r=j \neq 1 \text { and } s=i=1 \\ 0 & \text { if } r=s=i=j\end{cases}
$$

See the multiplication table in section 7. By abuse of notation we may think of this operation as

$$
U \cdot W^{m}=[U, W]^{m} .
$$

We extend the operation to all of $\mathfrak{g}_{f}$ by the rule 


$$
[a, b] \cdot v=a \cdot(b \cdot v)-(-1)^{|a||b|} b \cdot(a \cdot v)
$$

so $M$ is a $\mathfrak{g}_{f}$-module.

The Lie algebra $\mathfrak{g}$ is the quotient of $\mathfrak{g}_{f}$ by the ideal of relation REL so to show that $M$ is in fact a $\mathfrak{g}$-module it suffices to show $r \cdot v=0$ for any $r \in$ Rel and any $v \in M$. This can be done by direct calculations which are given in appendix 1 .

To use the theory of [2] we extend our Lie algebra $\mathfrak{g}$ to a Lie algebra $\mathfrak{g}^{\sharp}=k \mathbf{1} \oplus \mathfrak{g}$ where $\mathbf{1}$ is an even symbol of degree 0 and the bracket on $\mathfrak{g}$ is extended to $\mathfrak{g}^{\sharp}$ by the rule $[g, \mathbf{1}]=\operatorname{deg}(g) g$ for $g \in \mathfrak{g}$. This defines a Lie structure on $\mathfrak{g}^{\sharp}$ since $g \longrightarrow \operatorname{deg}(g) g$ is a derivation. We make $M$ into a $\mathfrak{g}^{\sharp}$ module by defining

$$
1 . v=-\operatorname{deg}(v) v \quad \text { for any } v \in M
$$

By definition rule (7) holds for any $a, b$ with $\operatorname{deg} a, \operatorname{deg} b \geq 1$ so we only need to check that $[a, \mathbf{1}] . v=a .(\mathbf{1} . v)-\mathbf{1} .(a . v)$ holds for any $a \in \mathfrak{g}$ and any $v \in M$. We get

$$
\text { a. }(\mathbf{1} . v)-1 .(a . v)=-\operatorname{deg}(v) a \cdot v-(-\operatorname{deg}(a . v)) a \cdot v=\operatorname{deg}(a) a . v
$$

By definition $[a, \mathbf{1}]=\operatorname{deg}(a) a$ so the rule holds. Hence $M$ is a $\mathfrak{g}^{\sharp}$-module

\subsection{The map}

We define a map of graded $\mathfrak{g}^{\sharp}$-modules $M \longrightarrow \mathfrak{g}^{\sharp}$ such that $f\left(m_{0}\right)=\mathbf{1}$ and $f\left(w^{m}\right)=w$ for $w^{m}$ a generator of $M_{\geq 1}$. By construction $f$ is surjective and by theorem 5.4 in [2] we have that $\operatorname{ker}(f)$ is generated by

$$
\left\{f(a) \cdot m_{0}-\operatorname{deg}(a) a ; a \in M_{\geq 1}\right\} .
$$

We will show that for any $a \in M_{\geq 1}$ we have

$$
f(a) \cdot m_{0}=\operatorname{deg}(a) a
$$

i.e. $f$ is injective.

Degree 1: We have

$$
f\left(X_{l k}\right) \cdot m_{0}=X_{l k} \cdot m_{0}=X_{l k}^{m}
$$

and similarly for $Y_{l k}^{m}$ and $Z_{l k}^{m}$.

Degree 2: We write $a_{i j}=\left[X_{i r}, Y_{r j}\right]$. 


$$
\begin{aligned}
f\left(a_{i j}^{m}\right) \cdot m_{0} & =a_{i j} \cdot m_{0} \\
& =\left[X_{i r}, Y_{r j}\right] \cdot m_{0} \\
& =X_{i r} \cdot\left(Y_{r j} \cdot m_{0}\right)+Y_{r j} \cdot\left(X_{i r} \cdot m_{0}\right) \\
& =X_{i r} \cdot Y_{r j}^{m}+Y_{r j} \cdot X_{i r}^{m} \\
& =a_{i j}^{m}+a_{i j}^{m} \\
& =2 a_{i j}^{m} \cdot \\
f\left(\alpha_{k}^{m}\right) \cdot m_{0} & =\alpha_{k} \cdot m_{0} \\
& =\left[X_{1 k}, Y_{k 1}\right] \cdot m_{0} \\
& =X_{1 k} \cdot\left(Y_{k 1} \cdot m_{0}\right)+Y_{k 1} \cdot\left(X_{1 k} \cdot m_{0}\right) \\
& =X_{1 k} \cdot Y_{k 1}^{m}+Y_{k 1} \cdot X_{1 k}^{m} \\
& =\alpha_{k}^{m}+\alpha_{k}^{m} \\
& =2 \alpha_{k}^{m} .
\end{aligned}
$$

Symmetry gives the result for $b_{i j}^{m}, \beta_{k}^{m}, d_{i j}^{m}$ and $\delta_{k}^{m}$.

Degree 3: We write $\Gamma_{i s}=\left[Z_{i p}, a_{p s}\right]$ with $p \neq s$ and $a_{p s}=\left[X_{p r}, Y_{r s}\right]$ with $r \neq i$ and $r \neq p$. We have

$$
\begin{aligned}
f\left(\Gamma_{i s}^{m}\right) \cdot m_{0} & =\Gamma_{i s} \cdot m_{0} \\
& =\left[Z_{i p}, a_{p s}\right] \cdot m_{0} \\
& =Z_{i p} \cdot\left(a_{p s} \cdot m_{0}\right)-a_{p s} \cdot\left(Z_{i p} \cdot m_{0}\right) \\
& =2 Z_{i p} \cdot a_{p s}^{m}-a_{p s} \cdot Z_{i p}^{m} \\
& =2 \Gamma_{i s}^{m}-X_{p r} \cdot\left(Y_{r s} \cdot Z_{i p}^{m}\right)-Y_{r s} \cdot\left(X_{p r} \cdot Z_{i p}^{m}\right) \\
& =2 \Gamma_{i s}^{m}-X_{p r} \cdot 0+Y_{r s} \cdot b_{i r}^{m} \\
& =2 \Gamma_{i s}^{m}+\Gamma_{i s}^{m} \\
& =3 \Gamma_{i s}^{m}
\end{aligned}
$$

Write $H=\left[Z_{11},\left[X_{12}, Y_{21}\right]\right]$.

$$
\begin{aligned}
f\left(H^{m}\right) \cdot m_{0} & =H \cdot m_{0} \\
& =\left[Z_{11},\left[X_{12}, Y_{21}\right]\right] \cdot m_{0} \\
& =Z_{11}\left(\left[X_{12}, Y_{21}\right] \cdot m_{0}\right)-\left[X_{12}, Y_{21}\right] \cdot\left(Z_{11} \cdot m_{0}\right) \\
& =2 Z_{11} \cdot \alpha_{2}^{m}-\left(X_{12} \cdot\left(Y_{21} \cdot Z_{11}^{m}\right)+Y_{21} \cdot\left(X_{12} \cdot Z_{11}^{m}\right)\right) \\
& =2 H^{m}-X_{12} \cdot d_{21}^{m}+Y_{21} \cdot b_{12}^{m} \\
& =2 H^{m}-F^{m}+H^{m}-H_{12}^{m}+F^{m}+H_{12}^{m} \\
& =3 H^{m}
\end{aligned}
$$


Write $a_{i 1}=\left[X_{i r}, Y_{r 1}\right]$ with $r \neq i, r \neq 1$

$$
\begin{aligned}
f\left(H_{1 i}^{m}\right) \cdot m_{0} & =H_{1 i} \cdot m_{0} \\
& =\left[Z_{1 i}, a_{i 1}\right] \cdot m_{0} \\
& =Z_{1 i} \cdot\left(a_{i 1} \cdot m_{0}\right)-a_{i 1} \cdot\left(Z_{1 i} \cdot m_{0}\right) \\
& =2 Z_{1 i} \cdot a_{i 1}^{m}-\left[X_{i r}, Y_{r 1}\right] \cdot Z_{1 i}^{m} \\
& =2 H_{1 i}^{m}-\left(X_{i r} \cdot\left(Y_{r 1} \cdot Z_{1 i}^{m}\right)+Y_{r 1} \cdot\left(X_{i r} \cdot Z_{1 i}^{m}\right)\right) \\
& =2 H_{1 i}^{m}-X_{i r} \cdot d_{r i}^{m}+Y_{r 1} \cdot b_{1 r}^{m} \\
& =2 H_{1 i}^{m}-F^{m}-H_{1 r}^{m}+H_{1 i}^{m}+F^{m}+H_{1 r}^{m} \\
& =3 H_{1 i}^{m}
\end{aligned}
$$

Write $F=\left[X_{11},\left[Y_{12}, Z_{21}\right]\right]$

$$
\begin{aligned}
f\left(F^{m}\right) \cdot m_{0} & =F \cdot m_{0} \\
& =\left[X_{11},\left[Y_{12}, Z_{21}\right]\right] \cdot m_{0} \\
& =X_{11} \cdot\left(\left[Y_{12}, Z_{21}\right] \cdot m_{0}\right)-\left[Y_{12}, Z_{21}\right] \cdot\left(X_{11} \cdot m_{0}\right) \\
& =2 X_{11} \cdot \delta_{2}^{m}+Y_{12} \cdot b_{21}^{m}-Z_{21} \cdot a_{12}^{m} \\
& =2 F^{m}+F^{m}+2 H^{m}-H_{12}^{m}-2 H^{m}+H_{12}^{m} \\
& =3 F^{m} .
\end{aligned}
$$

Write $\Upsilon_{X}=\left[X_{i j}, a_{j i}\right]$ and $a_{j i}=\left[X_{j r}, Y_{r i}\right]$ with $r \neq j$.

$$
\begin{aligned}
f\left(\Upsilon_{X}^{m}\right) \cdot m_{0} & =\Upsilon_{X} \cdot m_{0} \\
& =\left[X_{i j}, a_{j i}\right] \cdot m_{0} \\
& =X_{i j} \cdot\left(a_{j i} \cdot m_{0}\right)-a_{j i} \cdot\left(X_{i j} \cdot m_{0}\right) \\
& =2 X_{i j} \cdot a_{j i}-\left[X_{j r}, Y_{r i}\right] \cdot X_{i j}^{m} \\
& =2 Y_{X}^{m}-\left(X_{j r} \cdot\left(Y_{r i} \cdot X_{i j}^{m}\right)+Y_{r i} \cdot\left(X_{j r} \cdot X_{i j}^{m}\right)\right) \\
& =2 Y_{X}^{m}+X_{j r} \cdot a_{r j}^{m} \\
& =3 Y_{X}^{m}
\end{aligned}
$$

The calculations are similar for $\Upsilon_{Y}^{m}, \Xi_{X}^{m}, \Xi_{Z}^{m}, \Lambda_{Y}^{m}, \Lambda_{Z}^{m}$.

Degree 4: Write $\Phi_{X}=\left[X_{j i}, \Gamma_{i j}\right]$ and $\Gamma_{i j}=\left[Z_{i p}, a_{p j}\right]$ with $p \neq j, p \neq i$ and $a_{p j}=\left[X_{p r}, Y_{r j}\right]$ with $r \neq p, r \neq j$. We get 


$$
\begin{aligned}
f\left(\Phi_{X}^{m}\right) \cdot m_{0} & =\Phi_{X} \cdot m_{0} \\
& =X_{j i} \cdot\left(\Gamma_{i j} \cdot m_{0}\right)+\Gamma_{i j} \cdot\left(X_{j i} \cdot m_{0}\right) \\
& =3 X_{j i} \cdot \Gamma_{i j}^{m}+\left[Z_{i p}, a_{p j}\right] \cdot X_{j i}^{m} \\
& =3 \Phi_{X}^{m}+Z_{i p} \cdot\left(a_{p j} \cdot X_{j i}^{m}\right)-a_{p j} \cdot\left(Z_{i p} \cdot X_{j i}^{m}\right) \\
& =3 \Phi_{X}^{m}+Z_{i p} \cdot 0-a_{p j} \cdot b_{j p}^{m} \\
& =3 \Phi_{X}^{m}-\left(X_{p r} \cdot\left(Y_{r j} \cdot b_{j p}^{m}\right)+Y_{r j} \cdot\left(X_{p r} \cdot b_{j p}^{m}\right)\right) \\
& =3 \Phi_{X}^{m}+X_{p r} \cdot \Gamma_{r p}^{m}+Y_{r j} \cdot 0 \\
& =3 \Phi_{X}^{m}+\Phi_{X}^{m} \\
& =4 \Phi_{X}^{m}
\end{aligned}
$$

The calculations are similar for $\Phi_{Y}$ and $\Phi_{Z}$.

\subsection{Conclusion}

We have proved that $f$ is injective and hence an isomorphism. This implies that the generating set for $\mathfrak{g}$ is a basis so $\operatorname{dim} \mathfrak{g}_{1}=3 n^{2}, \operatorname{dim} \mathfrak{g}_{2}=3\left(n^{2}-1\right)$, $\operatorname{dim} \mathfrak{g}_{3}=n^{2}+7, \operatorname{dim} \mathfrak{g}_{4}=3$ and $\mathfrak{g}_{5}=\mathfrak{g}_{6}=\cdots=0$. This gives that the Hilbert series of $R^{!}$is

$$
H_{R^{!}}(t)=\frac{(1+t)^{3 n^{2}}\left(1+t^{3}\right)^{n^{2}+7}}{\left(1-t^{2}\right)^{3\left(n^{2}-1\right)}\left(1-t^{4}\right)^{3}}
$$

\section{Appendix 1}

\subsection{Degree 0}

We start by checking that the relations give zero when operating on $m_{0}$. Let $U$ and $V$ be two variables in the Lie algebra. Then

$$
[U, V] \cdot m_{0}=U \cdot\left(V \cdot m_{0}\right)+V \cdot\left(U \cdot m_{0}\right)=U \cdot V^{m}+V \cdot U^{m} .
$$

Since the operation of the Lie algebra on the module is defined similarly to the Lie algebra multiplication for elements of degree 1 we have that if $[U, V]=0$ in the Lie algebra then $U . V^{m}=0$ and $V \cdot U^{m}=0$ so $[U, V] \cdot m_{0}=0$. So all the relations that are given by a single Lie monomial give 0 when operating on $m_{0}$.

Now we look at the relation

$$
\left[X_{i r}, Y_{r j}\right]-\left[X_{i s}, Y_{s j}\right] \quad \text { where } i \neq j, r, s, \in\{1, \ldots, n\} .
$$

We get

$$
\begin{aligned}
{\left[X_{i k}, Y_{k j}\right] \cdot m_{0} } & =X_{i k} \cdot\left(Y_{k j} \cdot m_{0}\right)+Y_{k j} \cdot\left(X_{i k} \cdot m_{0}\right)=X_{i k} \cdot Y_{k j}^{m}+Y_{k j} \cdot X_{i k}^{m} \\
& =a_{i j}^{m}+a_{i j}^{m}=2 a_{i j}^{m} \quad \text { for any } k \in\{1, \ldots, n\} .
\end{aligned}
$$


So

$$
\left(\left[X_{i r}, Y_{r j}\right]-\left[X_{i s}, Y_{s j}\right]\right) \cdot m_{0}=0 .
$$

We also get for any $k \in\{1, \ldots, n\}$

$$
\left[X_{k j}, Y_{i k}\right] \cdot m_{0}=X_{k j} \cdot Y_{i k}^{m}+Y_{i k} \cdot X_{k j}^{m}=-2 a_{i j}^{m} .
$$

So

$$
\left(\left[X_{i r}, Y_{r j}\right]+\left[X_{r j}, Y_{i r}\right]\right) \cdot m_{0}=0
$$

There are two more relations in $X$ and $Y$ that we need to check:

$$
\begin{aligned}
{\left[X_{i j}, Y_{j i}\right]+\left[X_{j i}, Y_{i j}\right]=0 } & \text { for } i, j \in\{1, \ldots, n\} \\
{\left[X_{1 i}, Y_{i 1}\right]-\left[X_{1 j}, Y_{j 1}\right]+\left[X_{i j}, Y_{j i}\right]=0 } & \text { for } i, j \in\{2, \ldots, n\} .
\end{aligned}
$$

We get:

$$
\begin{aligned}
{\left[X_{i j}, Y_{j i}\right] \cdot m_{0} } & =X_{i j} \cdot\left(Y_{j i} \cdot m_{0}\right)+Y_{j i} \cdot\left(X_{i j} \cdot m_{0}\right)=X_{i j} \cdot Y_{j i}^{m}+Y_{j i} \cdot X_{i j}^{m} \\
& = \begin{cases}2\left(\alpha_{j}^{m}-\alpha_{i}^{m}\right) & \text { if } i \neq 1 \text { and } j \neq 1 \\
2 \alpha_{j}^{m} & \text { if } i=1 \\
-2 \alpha_{i}^{m} & \text { if } j=1\end{cases}
\end{aligned}
$$

and

$$
\begin{aligned}
{\left[X_{j i}, Y_{i j}\right] . m_{0} } & =X_{j i} \cdot\left(Y_{i j} \cdot m_{0}\right)+Y_{i j} \cdot\left(X_{j i} \cdot m_{0}\right)=X_{j i} \cdot Y_{i j}^{m}+Y_{i j} \cdot X_{j i}^{m} \\
& = \begin{cases}2\left(\alpha_{i}^{m}-\alpha_{j}^{m}\right) & \text { if } i \neq 1 \text { and } j \neq 1 \\
-2 \alpha_{j}^{m} & \text { if } i=1 \\
2 \alpha_{i}^{m} & \text { if } j=1\end{cases}
\end{aligned}
$$

so

$$
\left(\left[X_{i j}, Y_{j i}\right]+\left[X_{j i}, Y_{i j}\right]\right) \cdot m_{0}=0
$$

and

$$
\left(\left[X_{1 i}, Y_{i 1}\right]-\left[X_{1 j}, Y_{j 1}\right]+\left[X_{i j}, Y_{j i}\right]\right) \cdot m_{0}=2 \alpha_{i}^{m}-2 \alpha_{j}^{m}+2\left(\alpha_{j}^{m}-\alpha_{i}^{m}\right)=0 .
$$

Similarly we get that the relations of degrees $(1,0,1)$ and $(0,1,1)$ give zeros when they operate on $m_{0}$.

\subsection{Degree 1}

To check that the relations give 0 when operating on degree 1 in the module it suffices to check this on $X_{k l}^{m}$ for any $k$ and $l$. For symmetry reasons we get the same results for $Y_{k l}^{m}$ and $Z_{k l}^{m}$. 
We have that the multidegree of $\left[X_{i j}, X_{r s}\right] . X_{k l}^{m}$ is $(3,0,0)$ and as there are no elements in the module of this multidegree this has to be zero.

We now consider the relations of degree $(0,2,0)$. We have

$$
\left[Y_{i j}, Y_{r s}\right] \cdot X_{l k}^{m}=Y_{i j} \cdot\left(Y_{r s} \cdot X_{l k}^{m}\right)+Y_{r s} \cdot\left(Y_{i j} \cdot X_{l k}^{m}\right)
$$

and

$$
Y_{r s} . X_{l k}^{m}= \begin{cases}0 & \text { if } s \neq l \text { and } r \neq k \\ a_{l s}^{m} & \text { if } r=k \text { and } s \neq l \\ -a_{r k}^{m} & \text { if } r \neq k \text { and } s=l \\ \alpha_{k}^{m}-\alpha_{l}^{m} & \text { if } r=k \neq 1 \text { and } s=l \neq 1 \text { and } l \neq k \\ \alpha_{k}^{m} & \text { if } r=k \neq 1 \text { and } s=l=1 \\ -\alpha_{l}^{m} & \text { if } r=k=1 \text { and } s=l \neq 1 \\ 0 & \text { if } r=s=k=l\end{cases}
$$

so

$$
Y_{i j} \cdot\left(Y_{r s} . X_{l k}^{m}\right)= \begin{cases}\Upsilon_{Y}^{m} & \text { if } j=l, i=s, r=k, i \neq j \\ -\Upsilon_{Y}^{m} & \text { if } j=r, i=k, s=l, i \neq j \\ \Upsilon_{Y}^{m} & \text { if } i=j=s=l, r=k \neq i \\ \Upsilon_{Y}^{m} & \text { if } i=j=r=k, s=l \neq i \\ 0 & \text { otherwise }\end{cases}
$$

In the nonzero cases the second term is

$$
Y_{r s} \cdot\left(Y_{i j} \cdot X_{l k}^{m}\right)=\left\{\begin{array}{l}
Y_{k s} \cdot\left(Y_{s l} \cdot X_{l k}^{m}\right)=-\Upsilon_{Y}^{m} \\
Y_{r l} \cdot\left(Y_{k r} \cdot X_{l k}^{m}\right)=\Upsilon_{Y}^{m} \\
Y_{k l} \cdot\left(Y_{l l} \cdot X_{l k}^{m}\right)=-\Upsilon_{Y}^{m} \\
Y_{k l} \cdot\left(Y_{k k} \cdot X_{l k}^{m}\right)=-\Upsilon_{Y}^{m}
\end{array}\right.
$$

so the two terms cancel. Similarly we get the same result if we start by examining the nonzero cases for the second term. So

$$
\left[Y_{i j}, Y_{r s}\right] \cdot X_{l k}^{m}=0 \quad \text { for any } i, j, r, s, l, k
$$

By symmetry we get that the relations

$$
\left[Z_{i j}, Z_{r s}\right]=0 \text { for all } i, j, r, s \in\{1, \ldots, n\} .
$$

give zero when operating on $X_{l k}^{m}$.

We now consider the relations of degree $(1,1,0)$. We start with

$$
\begin{array}{ll}
{\left[X_{i j}, Y_{r s}\right]=0} & \text { if } j \neq r \text { and } i \neq s \\
{\left[X_{i j}, Y_{i j}\right]=0} & \text { for all }(i, j)
\end{array}
$$

We get

$$
\left[X_{i j}, Y_{r s}\right] \cdot X_{l k}^{m}=X_{i j} \cdot\left(Y_{r s} \cdot X_{l k}^{m}\right)+Y_{r s} \cdot\left(X_{i j} \cdot X_{l k}^{m}\right)
$$


The last term is zero. In (8) we have the different possibilities for $\left(Y_{r s} . X_{l k}^{m}\right)$ so we get

$$
X_{i j} \cdot\left(Y_{r s} \cdot X_{l k}^{m}\right)=\left\{\begin{array}{lll}
X_{i j} \cdot a_{l s}^{m} & =0 & \text { if } i \neq s \\
-X_{i j} \cdot a_{r k}^{m} & =0 & \text { if } j \neq r \\
X_{i j} \cdot\left(\alpha_{r}^{m}-\alpha_{s}^{m}\right)=0 & \text { if } i \neq s \text { and } j \neq r \\
X_{i j} \cdot \alpha_{r}^{m}=0 & \text { if } j \neq r \\
-X_{i j} \cdot \alpha_{s}^{m} & =0 & \text { if } i \neq s \\
0 & & \text { otherwise }
\end{array}\right.
$$

From this we get

$$
\begin{array}{ll}
{\left[X_{i j}, Y_{r s}\right] \cdot X_{l k}^{m}=0} & \text { if } i \neq s \text { and } j \neq r \\
{\left[X_{i j}, Y_{i j}\right] \cdot X_{l k}^{m}=0} & \text { if } i \neq j
\end{array}
$$

and

$$
\left[X_{i i}, Y_{i i}\right] \cdot X_{l k}^{m}=X_{i i} \cdot\left\{\begin{array}{ll}
a_{l i}^{m} & \text { if } i=k \neq l \\
-a_{i k}^{m} & \text { if } i=l \neq k \\
0 & \text { otherwise }
\end{array}\right\}=0
$$

Now we consider the relations

$$
\begin{aligned}
& {\left[X_{i r}, Y_{r j}\right]-\left[X_{i s}, Y_{s j}\right]=0 \quad r, s, i, j \in\{1, \ldots, n\}, i \neq j} \\
& {\left[X_{i r}, Y_{r j}\right]+\left[X_{r j}, Y_{i r}\right]=0 \quad r, i, j \in\{1, \ldots, n\}, i \neq j} \\
& {\left[X_{i j}, Y_{j i}\right]+\left[X_{j i}, Y_{i j}\right]=0 \quad i, j \in\{1, \ldots, n\}} \\
& {\left[X_{1 i}, Y_{i 1}\right]-\left[X_{1 j}, Y_{j 1}\right]+\left[X_{i j}, Y_{j i}\right]=0 \quad i, j \in\{2, \ldots, n\} .}
\end{aligned}
$$

First we calculate $\left[X_{i q}, Y_{q j}\right] . X_{l k}^{m}$ for some $q \in\{1, \ldots, n\}$.

$$
\left[X_{i q}, Y_{q j}\right] \cdot X_{l k}^{m}=X_{i q} \cdot\left(Y_{q j} \cdot X_{l k}^{m}\right)+Y_{q j} \cdot\left(X_{i q} \cdot X_{l k}^{m}\right)
$$

The last term is zero. Using (8) we get

(9) $\quad X_{i q} \cdot\left(Y_{q j} \cdot X_{l k}^{m}\right)=X_{i q} \cdot\left\{\begin{array}{ll}a_{l j}^{m} & \text { if } q=k \text { and } j \neq l \\ -a_{q k}^{m} & \text { if } q \neq k \text { and } j=l \\ \alpha_{k}^{m}-\alpha_{l}^{m} & \text { if } q=k \neq 1 \text { and } j=l \neq 1 \\ \alpha_{k}^{m} & \text { if } q=k \neq 1 \text { and } j=l=1 \\ -\alpha_{l}^{m} & \text { if } q=k=1 \text { and } j=l \neq 1 \\ 0 & \text { otherwise }\end{array}\right\}$ 


$$
= \begin{cases}\Upsilon_{X}^{m} & \text { if } i=j \neq l \text { and } q=l=k \\ -\Upsilon_{X}^{m} & \text { if } i=k \neq q \text { and } j=l \\ -\Upsilon_{X}^{m} & \text { if } i=k=q \neq 1, j=l \neq 1 \text { and } i \neq j \\ -\Upsilon_{X}^{m} & \text { if } i=k=q \neq 1 \text { and } j=l=1 \\ -\Upsilon_{X}^{m} & \text { if } i=k=q=1 \text { and } j=l \neq 1 \\ 0 & \text { otherwise }\end{cases}
$$

So for $i \neq j$ we have

$$
X_{i q} \cdot\left(Y_{q j} \cdot X_{l k}^{m}\right)= \begin{cases}-\Upsilon_{X}^{m} & \text { if } i=k \text { and } j=l \\ 0 & \text { otherwise }\end{cases}
$$

i.e. this is independent of $q$ so the relation

$$
\left[X_{i r}, Y_{r j}\right]-\left[X_{i s}, Y_{s j}\right] \quad \text { with } i \neq j
$$

gives 0 when operating on $X_{l k}^{m}$.

Similar calculations give for $i \neq j$

$$
X_{q i} \cdot\left(Y_{j q} \cdot X_{l k}^{m}\right)= \begin{cases}Y_{X}^{m} & \text { if } i=l \text { and } k=j \\ 0 & \text { otherwise }\end{cases}
$$

So the relation

$$
\left[X_{i r}, Y_{r j}\right]+\left[X_{r j}, Y_{i r}\right]=0
$$

gives 0 when operating on $X_{l k}^{m}$.

Equation (9) also gives us:

$$
X_{i q \cdot}\left(Y_{q i} \cdot X_{l k}^{m}\right)= \begin{cases}\Upsilon_{X}^{m} & \text { if } k=q=l \neq i \\ -\Upsilon_{X}^{m} & \text { if } k=i=l \neq q \\ 0 & \text { otherwise }\end{cases}
$$

and similar calculations give

$$
X_{q i} \cdot\left(Y_{i q} \cdot X_{l k}^{m}\right)= \begin{cases}-\Upsilon_{X}^{m} & \text { if } k=q=l \neq i \\ \Upsilon_{X}^{m} & \text { if } k=i=l \neq q \\ 0 & \text { otherwise }\end{cases}
$$

so the relation $\left[X_{i q}, Y_{q i}\right]+\left[X_{q i}, Y_{i q}\right]$ gives 0 when operating on $X_{l k}^{m}$.

Let $i, j \neq 1$ and $i \neq j$ then we get from equations (9) and (10): 


$$
\begin{aligned}
& \left(\left[X_{1 i}, Y_{i 1}\right]-\left[X_{1 j}, Y_{j 1}\right]+\left[X_{i j}, Y_{j i}\right]\right) \cdot X_{l k}^{m} \\
& =X_{1 i} \cdot\left(Y_{i 1} \cdot X_{l k}^{m}\right)-X_{1 j} \cdot\left(Y_{j 1} \cdot X_{l k}^{m}\right)+X_{i j} \cdot\left(Y_{j i} \cdot X_{l k}^{m}\right) \\
& \quad=\left\{\begin{array}{ll}
\Upsilon_{X}^{m} & \text { if } k=l=i \\
-\Upsilon_{X}^{m} & \text { if } k=l=1 \\
0 & \text { otherwise }
\end{array}\right\} \\
& \quad-\left\{\begin{array}{ll}
\Upsilon_{X}^{m} & \text { if } k=l=j \\
-\Upsilon_{X}^{m} & \text { if } k=l=1 \\
0 & \text { otherwise }
\end{array}\right\} \\
& +\left\{\begin{array}{ll}
\Upsilon_{X}^{m} & \text { if } k=l=j \\
-\Upsilon_{X}^{m} & \text { if } k=l=i \\
0 & \text { otherwise }
\end{array}\right\} \\
& =0
\end{aligned}
$$

We have now checked that all relations of multidegree $(1,1,0)$ give 0 when operating on $X_{l k}^{m}$. For the relations of multidegree $(1,0,1)$ the calculations are similar. What remains to be checked are the relations of degree $(0,1,1)$.

We get

Claim 15. If $i \neq s$ and $j \neq r$ then

$$
\left[Y_{i j}, Z_{r s}\right] \cdot X_{l k}^{m}=0
$$

Proof. We have

$$
\left[Y_{i j}, Z_{r s}\right] \cdot X_{l k}^{m}=Y_{i j} \cdot\left(Z_{r s} \cdot X_{l k}^{m}\right)+Z_{r s} \cdot\left(Y_{i j} \cdot X_{l k}^{m}\right)
$$

and $Z_{r s} . X_{l k}^{m}=0$ if $r \neq k$ and $s \neq l$ and $Y_{i j} . X_{l k}^{m}=0$ if $i \neq k$ and $j \neq l$. We now go through the different cases:

If $r=k$ and $s \neq l$ then (11) becomes

$$
Y_{i j} \cdot\left(Z_{k s} \cdot X_{l k}^{m}\right)+Z_{k s} \cdot\left(Y_{i j} \cdot X_{l k}^{m}\right)
$$

and we have the following subcases:



If $r \neq k$ and $s=l$ then (11) becomes

$$
Y_{i j} \cdot\left(Z_{r l} \cdot X_{l k}^{m}\right)+Z_{r l} \cdot\left(Y_{i j} \cdot X_{l k}^{m}\right)
$$


and we have the following subcases:

\begin{tabular}{|c|c|c|c|c|c|c|c|c|c|}
\hline subcases & $Y_{i j} \cdot\left(Z_{r l} \cdot X_{l k}^{m}\right)$ & + & $Z_{r l} \cdot\left(Y_{i j} \cdot X_{l k}^{m}\right)$ & & & & & & \\
\hline$i \neq k, j \neq l$ & $-Y_{i j} \cdot b_{r k}^{m}$ & + & 0 & $=$ & 0 & + & 0 & $=$ & 0 \\
\hline$i=k, j \neq l$ & $-Y_{k j} \cdot b_{r k}^{m}$ & + & $Z_{r l} \cdot a_{l j}^{m}$ & $=$ & $-\Gamma_{r j}^{m}$ & + & $\Gamma_{r j}^{m}$ & $=$ & 0 \\
\hline$i \neq k, j=l$ & $-Y_{i l} \cdot b_{r k}^{m}$ & - & $Z_{r l} \cdot a_{i k}^{m}$ & $=$ & 0 & + & 0 & $=$ & 0 \\
\hline$i=k, j=l$ & $-Y_{k l} \cdot b_{r k}^{m}$ & + & $Z_{r l} \cdot\left(Y_{k l} \cdot X_{l k}^{m}\right)$ & $=$ & $-\Gamma_{r l}^{m}$ & + & $\Gamma_{r l}^{m}$ & $=$ & 0 \\
\hline
\end{tabular}

If $r=k$ and $s=l$ then (11) becomes

$$
Y_{i j} \cdot\left(Z_{k l} \cdot X_{l k}^{m}\right)+Z_{k l} \cdot\left(Y_{i j} \cdot X_{l k}^{m}\right)
$$

and we have the following subcases:

\begin{tabular}{|c|c|c|c|c|c|c|c|c|}
\hline subcases & $Y_{i j} .\left(Z_{k l} . X_{l k}^{m}\right)$ & $+Z_{k l} \cdot\left(Y_{i j} \cdot X_{l k}^{m}\right)$ & & & & & & \\
\hline$i \neq k, j \neq l$ & 0 & +0 & $=$ & 0 & + & 0 & $=$ & 0 \\
\hline$i=k, j \neq l$ & $Y_{k j} \cdot\left(Z_{k l} \cdot X_{l k}^{m}\right)$ & $+Z_{k l} \cdot a_{l j}^{m}$ & $=$ & $-\Gamma_{k j}^{m}$ & + & $\Gamma_{k j}^{m}$ & $=$ & 0 \\
\hline$i \neq k, j=l$ & $Y_{i l} \cdot\left(Z_{k l} \cdot X_{l k}^{m}\right)$ & $-Z_{k l} \cdot a_{i k}^{m}$ & $=$ & $-\Gamma_{i l}^{m}$ & + & $\Gamma_{i l}^{m}$ & $=$ & 0 \\
\hline$i=k, j=l$ & $Y_{k l} \cdot\left(Z_{k l} \cdot X_{l k}^{m}\right)$ & $+Z_{k l} \cdot\left(Y_{k l} \cdot X_{l k}^{m}\right)$ & $=$ & $-2 \Gamma_{k l}^{m}$ & + & $2 \Gamma_{k l}^{m}$ & $=$ & 0 \\
\hline
\end{tabular}

If $r \neq k$ and $s \neq l$ then we have the following subcases:

\begin{tabular}{|c|c|c|c|c|c|c|c|c|}
\hline subcases & $Y_{i j} .\left(Z_{r s} . X_{l k}^{m}\right)$ & $+Z_{r s \cdot} \cdot\left(Y_{i j} \cdot X_{l k}^{m}\right)$ & & & & & & \\
\hline$i \neq k, j \neq l$ & 0 & +0 & $=$ & 0 & + & 0 & $=$ & 0 \\
\hline$i=k, j \neq l$ & 0 & $+Z_{r s} \cdot a_{l j}^{m}$ & $=$ & 0 & + & 0 & $=$ & 0 \\
\hline$i \neq k, j=l$ & 0 & $-Z_{r s} \cdot a_{i k}^{m}$ & $=$ & 0 & + & 0 & $=$ & 0 \\
\hline$i=k, j=l$ & 0 & $+Z_{r s \cdot} \cdot\left(Y_{k l} \cdot X_{l k}^{m}\right)$ & $=$ & 0 & + & 0 & $=$ & 0 \\
\hline
\end{tabular}

So we have proved the claim.

Claim 16. For any $(i, j)$ we have

$$
\left[Y_{i j}, Z_{i j}\right] . X_{l k}^{m}=0
$$

Proof. The previous claim gives that for $i \neq j$ we have $\left[Y_{i j}, Z_{i j}\right] . X_{l k}^{m}=0$ so we only need to check that $\left[Y_{i i}, Z_{i i}\right] . X_{l k}^{m}=0$.

$$
\left[Y_{i i}, Z_{i i}\right] \cdot X_{l k}^{m}=Y_{i i} \cdot\left(Z_{i i} \cdot X_{l k}^{m}\right)+Z_{i i} \cdot\left(Y_{i i} \cdot X_{l k}^{m}\right)
$$

Both terms are 0 if $i \neq l$ and $i \neq k$. We have the following cases: 


\begin{tabular}{|c|c|c|c|c|c|c|c|c|c|}
\hline cases & $Y_{i i} \cdot\left(Z_{i i} \cdot X_{l k}^{m}\right)$ & + & $Z_{i i} \cdot\left(Y_{i i} \cdot X_{l k}^{m}\right)$ & & & & & & \\
\hline$i=k \neq l$ & $Y_{k k} \cdot b_{l k}^{m}$ & + & $Z_{k k} \cdot a_{l k}^{m}$ & $=$ & $\Gamma_{l k}^{m}$ & + & $-\Gamma_{l k}^{m}$ & $=$ & 0 \\
\hline$i=l \neq k$ & $-Y_{l l} \cdot b_{l k}^{m}$ & + & $-Z_{l l} \cdot a_{l k}^{m}$ & $=$ & $\Gamma_{l k}^{m}$ & + & $-\Gamma_{l k}^{m}$ & $=$ & 0 \\
\hline$i=k=l$ & $Y_{l l} \cdot\left(Z_{l l} \cdot X_{l l}^{m}\right)$ & + & $Z_{l l} \cdot\left(Y_{l l} \cdot X_{l l}^{m}\right)$ & $=$ & 0 & + & 0 & $=$ & 0 \\
\hline
\end{tabular}

Claim 17. For $i \neq j$

$$
\left[Y_{i q}, Z_{q j}\right] . X_{l k}^{m}
$$

is independent of $q$ and

$$
\left(\left[Y_{i q}, Z_{q j}\right]+\left[Y_{q j} \cdot Z_{i q}\right]\right) \cdot X_{l k}^{m}=0
$$

ProOF.

$$
\left[Y_{i q}, Z_{q j}\right] \cdot X_{l k}^{m}=Y_{i q} \cdot\left(Z_{q j} \cdot X_{l k}^{m}\right)+Z_{q j} \cdot\left(Y_{i q} \cdot X_{l k}^{m}\right)
$$

and

$$
\left[Y_{q j}, Z_{i q}\right] \cdot X_{l k}^{m}=Y_{q j} \cdot\left(Z_{i q} \cdot X_{l k}^{m}\right)+Z_{i q} \cdot\left(Y_{q j} \cdot X_{l k}^{m}\right)
$$

\begin{tabular}{|c|c|c|c|c|c|c|c|c|}
\hline subcases & {$\left[Y_{i q}, Z_{q j}\right] \cdot X_{l k}^{m}$} & $+\left[Y_{q j}, Z_{i q}\right] . X_{l k}^{m}$ & & & & & & \\
\hline$q=k, q \neq l$ & $Y_{i k} \cdot b_{l j}^{m}$ & $+Z_{i k} \cdot a_{l j}^{m}$ & $=$ & 0 & + & 0 & $=$ & 0 \\
\hline$q \neq k, q=l$ & $-Z_{l j} \cdot a_{i k}^{m}$ & $+-Y_{l j} \cdot b_{i k}^{m}$ & $=$ & 0 & + & 0 & $=$ & 0 \\
\hline$q=k=l$ & $-\Gamma_{i j}^{m}+\Gamma_{i j}^{m}$ & $+-\Gamma_{i j}^{m}+\Gamma_{i j}^{m}$ & $=$ & 0 & + & 0 & $=$ & 0 \\
\hline
\end{tabular}

These are both 0 unless $q=k$ or $j=l$ or $i=k$ or $q=l$. Assume $j \neq l$ and $i \neq k$. We get the following subcases:

\begin{tabular}{|c|c|c|c|c|c|c|c|c|}
\hline subcases & {$\left[Y_{i q}, Z_{q l}\right] \cdot X_{l k}^{m}$} & $+\left[Y_{q l}, Z_{i q}\right] \cdot X_{l k}^{m}$ & & & & & & \\
\hline$q \neq k, q \neq l$ & $-Y_{i q} \cdot b_{q k}^{m}$ & $+\quad-Z_{i q} \cdot a_{q k}^{m}$ & $=$ & $\Gamma_{i k}^{m}$ & + & $-\Gamma_{i k}^{m}$ & $=$ & 0 \\
\hline$q=k, q \neq l$ & $Y_{i k} \cdot\left(Z_{k l} \cdot X_{l k}^{m}\right)$ & $+Z_{i k} \cdot\left(Y_{k l} \cdot X_{l k}^{m}\right)$ & $=$ & $\Gamma_{i k}^{m}$ & + & $-\Gamma_{i k}^{m}$ & $=$ & 0 \\
\hline$q \neq k, q=l$ & $-Y_{i l} \cdot b_{l k}^{m}$ & $+\quad-Z_{i l} \cdot a_{l k}^{m}$ & $=$ & $\Gamma_{i k}^{m}$ & + & $-\Gamma_{i k}^{m}$ & $=$ & 0 \\
\hline$q=k=l$ & $-Z_{k k} \cdot a_{i k}^{m}$ & $+\quad-Y_{l l} \cdot b_{i l}^{m}$ & $=$ & $\Gamma_{i k}^{m}$ & + & $-\Gamma_{i k}^{m}$ & $=$ & 0 \\
\hline
\end{tabular}

So we have shown $\left[Y_{i q}, Z_{q j}\right] \cdot X_{l k}^{m}=0$ and $\left[Y_{q j}, Z_{i q}\right] \cdot X_{l k}^{m}=0$ if $j \neq l$ and $i \neq k$ (independent of the value of $q$ ).

Assume now that $j=l$ and $i \neq k$ then we get the following subcases:

So we have shown that if $j=l$ and $i \neq k$ then $\left[Y_{i q}, Z_{q l}\right] \cdot X_{l k}^{m}=\Gamma_{i k}^{m}$ (independent of the value of $q)$ and $\left(\left[Y_{i q}, Z_{q l}\right]+\left[Y_{q l}, Z_{i q}\right]\right) \cdot X_{l k}^{m}=0$.

Assume $j \neq l$ and $i=k$ then by calculations as above we get

$$
\left[Y_{k q}, Z_{q j}\right] \cdot X_{l k}^{m}=-\Gamma_{l j}^{m} \quad \text { and } \quad\left[Y_{q j}, Z_{k q}\right] \cdot X_{l k}^{m}=\Gamma_{l j}^{m} .
$$


Assume $j=l$ and $i=k$ then $k \neq l$ since $i \neq j$. We get

$$
\left[Y_{k q}, Z_{q l}\right] \cdot X_{l k}^{m}=Y_{k q} \cdot\left(Z_{q l} \cdot X_{l k}^{m}\right)+Z_{q l} \cdot\left(Y_{k q} \cdot X_{l k}^{m}\right)
$$

Here we need to divide into several subcases and subcases of those.

Case $l=1$ :

\begin{tabular}{l|lll} 
subcases & $Y_{k q} \cdot\left(Z_{q 1} \cdot X_{1 k}^{m}\right)$ & $+Z_{q 1} \cdot\left(Y_{k q} \cdot X_{1 k}^{m}\right)$ & \\
\hline$q=1$ & $-F^{m}-H_{1 k}^{m}$ & $+H^{m}$ & $=-F^{m}-H_{1 k}^{m}+H^{m}$ \\
$q=k$ & $-F^{m}-H^{m}$ & $+2 H^{m}-H_{1 k}^{m}$ & $=-F^{m}-H_{1 k}^{m}+H^{m}$ \\
$q \neq 1, q \neq k$ & $-F^{m}-H^{m}+H_{1 q}^{m}-H_{1 k}^{m}+2 H^{m}-H_{1 q}^{m}$ & $=-F^{m}-H_{1 k}^{m}+H^{m}$
\end{tabular}

So we have shown that $\left[Y_{k q}, Z_{q 1}\right] \cdot X_{1 k}^{m}=-F^{m}-H_{1 k}^{m}+H^{m}$ independent of the value of $q$.

Case $k=1$ : Similar calculations show that

$$
\left[Y_{1 q}, Z_{q l}\right] \cdot X_{l 1}^{m}=-F^{m}-H^{m}+H_{1 l}^{m}
$$

independent of the value of $q$.

Case $k \neq 1$ and $l \neq 1$

\begin{tabular}{l|lll} 
subcases & $Y_{k q} \cdot\left(Z_{q l} \cdot X_{l k}^{m}\right)$ & $+Z_{q l} \cdot\left(Y_{k q} \cdot X_{l k}^{m}\right)$ & \\
\hline$q=1$ & $-F^{m}-H_{1 k}^{m}$ & $+H_{1 l}^{m}$ & $=-F^{m}-H_{1 k}^{m}+H_{1 l}^{m}$ \\
$q=k$ & $-F^{m}-H^{m}$ & $+H^{m}-H_{1 k}^{m}+H_{1 l}^{m}=-F^{m}-H_{1 k}^{m}+H_{1 l}^{m}$ \\
$q=l$ & $-F^{m}-H^{m}+H_{1 l}^{m}-H_{1 k}^{m}+H^{m}$ & $=-F^{m}-H_{1 k}^{m}+H_{1 l}^{m}$ \\
$q \neq 1, k, l$ & $-F^{m}-H^{m}+H_{1 q}^{m}-H_{1 k}^{m}+H^{m}-H_{1 q}^{m}+H_{1 l}^{m}=-F^{m}-H_{1 k}^{m}+H_{1 l}^{m}$
\end{tabular}

So when $k \neq 1$ and $l \neq 1$ we get

$$
\left[Y_{k q}, Z_{q l}\right] \cdot X_{l k}^{m}=-F^{m}-H_{1 k}^{m}+H_{1 l}^{m}
$$

independent of the value of $q$.

We summarize the tables above and get

$$
\left[Y_{k q}, Z_{q l}\right] \cdot X_{l k}^{m}= \begin{cases}-F^{m}-H_{1 k}^{m}+H_{1 l}^{m} & \text { if } k \neq 1 \text { and } l \neq 1 \\ -F^{m}-H_{1 k}^{m}+H^{m} & \text { if } k \neq 1 \text { and } l=1 \\ -F^{m}-H^{m}+H_{1 l}^{m} & \text { if } k=1 \text { and } l \neq 1\end{cases}
$$

Now look at $\left[Y_{q j}, Z_{i q}\right] . X_{l k}^{m}$ for $j=l$ and $i=k$,

$$
\left[Y_{q l} \cdot Z_{k q}\right] \cdot X_{l k}^{m}=Y_{q l} \cdot\left(Z_{k q} \cdot X_{l k}^{m}\right)+Z_{k q} \cdot\left(Y_{q l} \cdot X_{l k}^{m}\right)
$$

The cases to check are the same as for $\left[Y_{k q}, Z_{q l}\right] . X_{l k}^{m}$ and similar calculations give 


$$
\left[Y_{q l}, Z_{k q}\right] \cdot X_{l k}^{m}= \begin{cases}F^{m}+H_{1 k}^{m}-H_{1 l}^{m} & \text { if } k \neq 1 \text { and } l \neq 1 \\ F^{m}+H_{1 k}^{m}-H^{m} & \text { if } k \neq 1 \text { and } l=1 \\ F^{m}+H^{m}-H_{1 l}^{m} & \text { if } k=1 \text { and } l \neq 1\end{cases}
$$

So we have proved the claim.

A consequence of this is that

$$
\left(\left[Y_{i r}, Z_{r j}\right]-\left[Y_{i s}, Z_{s j}\right]\right) \cdot X_{l k}^{m}=0
$$

and

$$
\left(\left[Y_{i r}, Z_{r j}\right]+\left[Y_{r j}, Z_{i r}\right] \cdot X_{l k}^{m}=0\right.
$$

Claim 18. Let $i \neq j$ then

$$
\left(\left[Y_{i j}, Z_{j i}\right]+\left[Y_{j i}, Z_{i j}\right]\right) \cdot X_{l k}^{m}=0
$$

Proof. We have

$$
\left[Y_{i j}, Z_{j i}\right] \cdot X_{l k}^{m}=Y_{i j} \cdot\left(Z_{j i} \cdot X_{l k}^{m}\right)+Z_{j i} \cdot\left(Y_{i j} \cdot X_{l k}^{m}\right)
$$

and

$$
\left[Y_{j i}, Z_{i j}\right] \cdot X_{l k}^{m}=Y_{j i} \cdot\left(Z_{i j} \cdot X_{l k}^{m}\right)+Z_{i j} \cdot\left(Y_{j i} \cdot X_{l k}^{m}\right) .
$$

We see that they are both 0 unless $i=k$ or $i=l$ or $j=k$ or $j=l$.

We now go through the different cases.

\begin{tabular}{l|l|l|l} 
Cases & subcases & {$\left[Y_{i j}, Z_{j i}\right] . X_{l k}^{m}$} & {$\left[Y_{j i}, Z_{i j}\right] . X_{l k}^{m}$} \\
\hline$j=k, i \neq l$ & $j=l$ & $F^{m}$ & $-F^{m}$ \\
& $j \neq l$ & $\Gamma_{l k}^{m}$ & $-\Gamma_{l k}^{m}$ \\
\hline$j=k, i=l$ & & $2 \Gamma_{l k}^{m}$ & $-2 \Gamma_{l k}^{m}$ \\
\hline$j \neq k, i=l$ & $i=k$ & $-F^{m}$ & $F^{m}$ \\
& $i \neq k$ & $\Gamma_{l k}^{m}$ & $-\Gamma_{l k}^{m}$ \\
\hline$j \neq k, i \neq l$ & $i=k, j=l$ & $-2 \Gamma_{l k}^{m}$ & $2 \Gamma_{l k}^{m}$ \\
& $i=k, j \neq l$ & $-\Gamma_{l k}^{m}$ & $\Gamma_{l k}^{m}$ \\
& $i \neq k, j \neq l$ & $-\Gamma_{l k}^{m}$ & $\Gamma_{l k}^{m}$
\end{tabular}

So we have proved the claim.

We summarize the above 


$$
\left[Y_{i j}, Z_{j i}\right] . X_{l k}^{m}= \begin{cases}F^{m} & \text { if } l=k=j \\ \Gamma_{l k}^{m} & \text { if } j=k \neq l \text { and } i \neq l \\ 2 \Gamma_{l k}^{m} & \text { if } j=k \text { and } i=l \\ -F^{m} & \text { if } i=l=k \\ \Gamma_{l k}^{m} & \text { if } i=l \neq k \text { and } j \neq k \\ -2 \Gamma_{l k}^{m} & \text { if } i=k \text { and } j=l \\ -\Gamma_{l k}^{m} & \text { if } i=k \neq l \text { and } j \neq l \\ -\Gamma_{l k}^{m} & \text { if } i \neq k \text { and } j=l \neq k\end{cases}
$$

Claim 19. For $i \neq j$ we have

$$
\left(\left[Y_{1 i}, Z_{i 1}\right]-\left[Y_{1 j}, Z_{j 1}\right]+\left[Y_{i j}, Z_{j i}\right]\right) \cdot X_{l k}^{m}=0
$$

Proof. We use (14) for each of the terms and get that this is 0 .

\subsection{Degree 2}

We now check that the relations give 0 when operating on degree 2 in the module. For symmetry reasons it is enough to check this for $a_{i j}^{m}$ and $\alpha_{k}^{m}$. We have that

\section{Rel. deg $2 \subset \operatorname{deg} 4$.}

Degree 4 in the module is generated by $\Phi_{X}^{m}, \Phi_{Y}^{m}$ and $\Phi_{Z}^{m}$ which have multidegrees $(2,1,1),(1,2,1)$ and $(1,1,2)$ respectively. The elements $a_{i j}^{m}$ and $\alpha_{k}^{m}$ both have multidegree $(1,1,0)$ so if $U$ is of degree 2 and is such that $U . a_{i j}^{m} \neq 0$ (or $U . \alpha_{k}^{m} \neq 0$ ) then $U$ has to have multidegree $(1,0,1)$, or $(0,1,1)$ or $(0,0,2)$. So its enough to check the relations that have these multidegrees. We start with the relation $\left[Z_{i j}, Z_{r s}\right]$

$$
\left[Z_{i j}, Z_{r s}\right] \cdot a_{k l}^{m}=Z_{i j} \cdot\left(Z_{r s} \cdot a_{k l}^{m}\right)+Z_{r s} \cdot\left(Z_{i j} \cdot a_{k l}^{m}\right) .
$$

The first term is 0 if $r \neq l$ and $k \neq s$ and the second term is 0 if $i \neq l$ and $j \neq k$. In the following table we list the nonzero possibilities for the first term and calculate the corresponding second term

\begin{tabular}{l|l|l|l} 
cases & $Z_{i j} \cdot\left(Z_{r s} \cdot a_{k l}^{m}\right)$ & subcases & $Z_{r s} \cdot\left(Z_{i j} \cdot a_{k l}^{m}\right)$ \\
\hline$j=r \neq l=i, k=s$ & $Z_{r l} \cdot\left(Z_{r k} \cdot a_{k l}^{m}\right)=\Phi_{Z}^{m}$ & $r \neq k$ & $Z_{r k} \cdot\left(Z_{l r} \cdot a_{k l}^{m}\right)=Z_{r k} \cdot\left(-\Gamma_{k r}^{m}\right)=-\Phi_{Z}^{m}$ \\
& & $r=k$ & $Z_{k k} \cdot\left(Z_{l k} \cdot a_{k l}^{m}\right)=-\Phi_{Z}^{m}$ \\
\hline$i=s \neq k=j, r=l$ & $Z_{s k} \cdot\left(Z_{l s} \cdot a_{k l}^{m}\right)=-\Phi_{Z}^{m}$ & $s \neq l$ & $Z_{l s} \cdot\left(Z_{s k} \cdot a_{k l}^{m}\right)=Z_{l s} \cdot \Gamma_{s l}^{m}=\Phi_{Z}^{m}$ \\
& & $s=l$ & $Z_{l l} \cdot\left(Z_{l k} \cdot a_{k l}^{m}\right)=\Phi_{Z}^{m}$ \\
\hline$r=l=i=j, s=k$ & $Z_{l l} \cdot\left(Z_{l k} \cdot a_{k l}^{m}\right)=\Phi_{Z}^{m}$ & & $Z_{l k} \cdot\left(Z_{l l} \cdot a_{k l}^{m}\right)=Z_{l k} \cdot\left(-\Gamma_{k l}^{m}\right)=-\Phi_{Z}^{m}$ \\
\hline$r=l, s=k=i=j$ & $Z_{k k} \cdot\left(Z_{l k} \cdot a_{k l}^{m}\right)=-\Phi_{Z}^{m}$ & & $Z_{l k} \cdot\left(Z_{k k} \cdot a_{k l}^{m}\right)=Z_{l k} \cdot \Gamma_{k l}^{m}=\Phi_{Z}^{m}$
\end{tabular}

So we see that when the first term is nonzero it is cancelled by the second term. For symmetry reason we get the same result if we start with the second 
term so $\left[Z_{i j}, Z_{r s}\right] \cdot a_{k l}^{m}=0$. We now consider $\left[Z_{i j}, Z_{r s}\right] \cdot \alpha_{k}^{m}$. We get the following nonzero possibilities

\begin{tabular}{l|l|l} 
cases & $Z_{i j} \cdot\left(Z_{r s} \cdot \alpha_{k}^{m}\right)$ & $Z_{r s} \cdot\left(Z_{i j} \cdot \alpha_{k}^{m}\right)$ \\
\hline $1 \neq s=i \neq k, j=r=1$ & $Z_{s 1} \cdot\left(Z_{1 s} \cdot \alpha_{k}^{m}\right)=Z_{s 1} \cdot\left(-\Gamma_{1 s}^{m}\right)=-\Phi_{Z}^{m}$ & $Z_{1 s} \cdot\left(Z_{s 1} \cdot \alpha_{k}^{m}\right)=\Phi_{Z}^{m}$ \\
$1 \neq s=i \neq k, j=r=k$ & $Z_{s k} \cdot\left(Z_{k s} \cdot \alpha_{k}^{m}\right)=Z_{s k} \cdot \Gamma_{k s}^{m}=\Phi_{Z}^{m}$ & $Z_{k s} \cdot\left(Z_{s k} \cdot \alpha_{k}^{m}\right)=-\Phi_{Z}^{m}$ \\
$1 \neq r=j \neq k, i=s=1$ & $Z_{1 r} \cdot\left(Z_{r 1} \cdot \alpha_{k}^{m}\right)=Z_{1 r} \cdot \Gamma_{r 1}^{m}=\Phi_{Z}^{m}$ & $Z_{r 1} \cdot\left(Z_{1 r} \cdot \alpha_{k}^{m}\right)=-\Phi_{Z}^{m}$ \\
$1 \neq r=j \neq k, i=s=1$ & $Z_{k r} \cdot\left(Z_{r k} \cdot \alpha_{k}^{m}\right)=Z_{k r} \cdot\left(-\Gamma_{r k}^{m}\right)=-\Phi_{Z}^{m}$ & $Z_{r k} \cdot\left(Z_{k r} \cdot \alpha_{k}^{m}\right)=\Phi_{Z}^{m}$ \\
$i=s=k, j=r=1$ & $Z_{k 1} \cdot\left(Z_{1 k} \cdot \alpha_{k}^{m}\right)=-2 \Phi_{Z}^{m}$ & $Z_{1 k} \cdot\left(Z_{k 1} \cdot \alpha_{k}^{m}\right)=2 \Phi_{Z}^{m}$ \\
$i=s=1, j=r=k$ & $Z_{1 k} \cdot\left(Z_{k 1} \cdot \alpha_{k}^{m}\right)=2 \Phi_{Z}^{m}$ & $Z_{k 1} \cdot\left(Z_{1 k} \cdot \alpha_{k}^{m}\right)=-2 \Phi_{Z}^{m}$
\end{tabular}

So we see that the first and the second term cancel.

We now consider the relations of multidegree $(1,0,1)$. We have

$$
\left[X_{i j}, Z_{r s}\right] \cdot a_{k l}^{m}=X_{i j} \cdot\left(Z_{r s} \cdot a_{k l}^{m}\right)+Z_{r s} \cdot\left(X_{i j} \cdot a_{k l}^{m}\right)
$$

We have $X_{i j} \cdot a_{k l}^{m}=\Upsilon_{X}^{m}$ or $X_{i j} \cdot a_{k l}^{m}=0$ and $Z_{r s} \cdot \Upsilon_{X}^{m}=0$ so the last term is always 0 . Now consider the first term:

$$
\begin{gathered}
X_{i j} \cdot\left(Z_{r s} \cdot a_{k l}^{m}\right)=X_{i j} \cdot \begin{cases}\Gamma_{r l}^{m} & \text { if } s=k \text { and } r \neq l \\
-\Gamma_{k s}^{m} & \text { if } s \neq k \text { and } r=l \\
H^{m}-H_{1 l}^{m}+H_{1 k}^{m} & \text { if } s=k \neq 1 \text { and } r=l \neq 1 \\
H_{1 k}^{m} & \text { if } s=k \neq 1 \text { and } r=l=1 \\
2 H^{m}-H_{1 l}^{m} & \text { if } s=k=1 \text { and } r=l \neq 1 \\
0 & \text { otherwise }\end{cases} \\
= \begin{cases}\Phi_{X}^{m} & \text { if } i=l, r=j, s=k, r \neq l \\
-\Phi_{X}^{m} & \text { if } i=s, k=j, s \neq k, r=l \\
\Phi_{X}^{m} & \text { if } i=j=l=r, s=k \\
-\Phi_{X}^{m} & \text { if } i=j=k=s, r=l \\
0 & \text { otherwise }\end{cases}
\end{gathered}
$$

i.e. the only nonzero possibilities for $X_{i j} \cdot\left(Z_{r s} \cdot a_{k l}^{m}\right)$ are

$$
\begin{aligned}
& X_{l r} \cdot\left(Z_{r k} \cdot a_{k l}^{m}\right)=\Phi_{X}^{m} \\
& X_{s k} \cdot\left(Z_{l s} \cdot a_{k l}^{m}\right)=-\Phi_{X}^{m}
\end{aligned}
$$

The relations of degree $(1,0,1)$ are:

$$
\begin{aligned}
{\left[X_{i j}, Z_{r s}\right] } & =0 & & \text { if } j \neq r \text { and } i \neq s \\
{\left[X_{i j}, Z_{i j}\right] } & =0 & & \text { for any }(i, j) \\
{\left[X_{i r}, Z_{r j}\right]-\left[X_{i s}, Z_{s j}\right] } & =0 & & r, s, i, j \in\{1, \ldots, n\}, i \neq j \\
{\left[X_{i r}, Z_{r j}\right]+\left[X_{r j}, Z_{i r}\right] } & =0 & & r, i, j \in\{1, \ldots, n\}, i \neq j \\
{\left[X_{i j}, Z_{j i}\right]+\left[X_{j i}, Z_{i j}\right] } & =0 & & i, j \in\{1, \ldots, n\}
\end{aligned}
$$


and

$$
\left[X_{1 i}, Z_{i 1}\right]-\left[X_{1 j}, Z_{j 1}\right]+\left[X_{i j}, Z_{j i}\right]=0 \quad i, j \in\{2, \ldots, n\} .
$$

From (15) and (16) it follows that

$$
\begin{array}{ll}
{\left[X_{i j}, Z_{r s}\right] \cdot a_{k l}^{m}=0} & \text { if } j \neq r \text { and } i \neq s \\
{\left[X_{i j}, Z_{i j}\right] \cdot a_{k l}^{m}=0} & \text { for all }(i, j)
\end{array}
$$

and

$$
\left[X_{i q}, Z_{q j}\right] \cdot a_{k l}^{m}= \begin{cases}\Phi_{X}^{m} & \text { if } i=l \text { and } j=k \\ 0 & \text { otherwise }\end{cases}
$$

So

$$
\left(\left[X_{i r}, Z_{r j}\right]-\left[X_{i s}, Z_{s j}\right]\right) \cdot a_{k l}^{m}=0 \quad \text { where } i \neq j
$$

Equations (15) and (16) also give

$$
\left[X_{i j}, Z_{j i}\right] \cdot a_{k l}^{m}=0
$$

So

$$
\begin{array}{r}
\left(\left[X_{i j}, Z_{j i}\right]+\left[X_{j i}, Z_{i j}\right]\right) \cdot a_{k l}^{m}=0 \\
\left(\left[X_{1 i}, Z_{i 1}\right]-\left[X_{1 j}, Z_{j 1}\right]+\left[X_{i j}, Z_{j i}\right]\right) \cdot a_{k l}^{m}=0 .
\end{array}
$$

We also have

$$
\left[X_{q j}, Z_{i q}\right] \cdot a_{k l}^{m}= \begin{cases}-\Phi_{X}^{m} & \text { if } j=k \text { and } i=l \\ 0 & \text { otherwise }\end{cases}
$$

so

$$
\left(\left[X_{i r}, Z_{r j}\right]+\left[X_{r j}, Z_{i r}\right]\right) \cdot a_{k l}^{m}=0 \quad \text { for } i \neq j .
$$

We have now shown that all the relations of degree $(1,0,1)$ give 0 when operating on $a_{k l}^{m}$.

For $\alpha_{k}^{m}$ we get similarly that $Z_{r s} \cdot\left(X_{i j} \cdot \alpha_{k}^{m}\right)=0$ and the nonzero possibilities for $X_{i j}\left(Z_{r s} \cdot \alpha_{k}^{m}\right)$ are:

$$
\begin{array}{ll}
X_{s 1} \cdot\left(Z_{1 s} \cdot \alpha_{k}^{m}\right)=-\Phi_{X}^{m} & s \neq 1, s \neq k \\
X_{s k} \cdot\left(Z_{k s} \cdot \alpha_{k}^{m}\right)=\Phi_{X}^{m} & s \neq 1, s \neq k \\
X_{1 r} \cdot\left(Z_{r 1} \cdot \alpha_{k}^{m}\right)=\Phi_{X}^{m} & r \neq 1, r \neq k \\
X_{k r} \cdot\left(Z_{r k} \cdot \alpha_{k}^{m}\right)=-\Phi_{X}^{m} & r \neq 1, r \neq k \\
X_{k 1} \cdot\left(Z_{1 k} \cdot \alpha_{k}^{m}\right)=-2 \Phi_{X}^{m} & \\
X_{1 k} \cdot\left(Z_{k 1} \cdot \alpha_{k}^{m}\right)=2 \Phi_{X}^{m} . &
\end{array}
$$


This implies that $\left[X_{i j}, Z_{r s}\right] \cdot \alpha_{k}^{m}=0$ if $i \neq s$ or $j \neq r$ so the relations (17), (18), (19) and (20) give 0 when operating on $\alpha_{k}^{m}$. This also gives

$$
\begin{aligned}
{\left[X_{s 1}, Z_{1 s}\right] \cdot \alpha_{k}^{m} } & =-\left[X_{1 s}, Z_{s 1}\right] \cdot \alpha_{k}^{m} \\
{\left[X_{s k}, Z_{k s}\right] \cdot \alpha_{k}^{m} } & =-\left[X_{k s}, Z_{s k}\right] \cdot \alpha_{k}^{m} \\
{\left[X_{k 1}, Z_{1 k}\right] \cdot \alpha_{k}^{m} } & =-\left[X_{1 k}, Z_{k 1}\right] \cdot \alpha_{k}^{m}
\end{aligned}
$$

so

$$
\left(\left[X_{i j}, Z_{j i}\right]+\left[X_{j i}, Z_{i j}\right]\right) \cdot \alpha_{k}^{m}=0
$$

and finally

$$
\begin{aligned}
\left(\left[X_{1 i}, Z_{i 1}\right]-\left[X_{1 j}, Z_{1 j}\right]+\left[X_{i j}, Z_{j i}\right]\right) \cdot \alpha_{k}^{m} & =\left\{\begin{array}{ll}
\Phi_{X}^{m} & \text { if } i \neq k \\
2 \Phi_{X}^{m} & \text { if } i=k
\end{array}\right\} \\
& -\left\{\begin{array}{ll}
\Phi_{X}^{m} & \text { if } j \neq k \\
2 \Phi_{X}^{m} & \text { if } j=k
\end{array}\right\} \\
& +\left\{\begin{array}{ll}
\Phi_{X}^{m} & \text { if } j=k \\
-\Phi_{X}^{m} & \text { if } i=k \\
0 & \text { otherwise }
\end{array}\right\} \\
& =0
\end{aligned}
$$

We have now shown that all the relations of degree $(1,0,1)$ give 0 when operating on $\alpha_{k}^{m}$.

For relations of degree $(0,1,1)$ the calculations are similar i.e. instead of $X_{i j} .\left(Z_{r s} \cdot a_{k l}^{m}\right)$ we have $Y_{i j} .\left(Z_{r s} \cdot a_{k l}^{m}\right)$ which behaves similarly.

\subsection{Degree 3 and 4}

Since Rel. deg $3 \subset \operatorname{deg} 5$ and Rel. deg $4 \subset \operatorname{deg} 6$ and there are no elements of degree 5 or higher in the module we have that the relations give 0 when operating on deg 3 and deg 4 . Hence we have shown that the relations give 0 when operating on $M$. 


\section{Appendix 2}

In this appendix we give the multiplication table for the case $n=3$.

Degree 2

Degree $(1,1,0)$.

\begin{tabular}{l|rrrrrrrrr} 
& $Y_{11}$ & $Y_{12}$ & $Y_{13}$ & $Y_{21}$ & $Y_{22}$ & $Y_{23}$ & $Y_{31}$ & $Y_{32}$ & $Y_{33}$ \\
\hline$X_{11}$ & 0 & $a_{12}$ & $a_{13}$ & $-a_{21}$ & 0 & 0 & $-a_{31}$ & 0 & 0 \\
$X_{12}$ & $-a_{12}$ & 0 & 0 & $\alpha_{2}$ & $a_{12}$ & $a_{13}$ & $-a_{32}$ & 0 & 0 \\
$X_{13}$ & $-a_{13}$ & 0 & 0 & $-a_{23}$ & 0 & 0 & $\alpha_{3}$ & $a_{12}$ & $a_{13}$ \\
$X_{21}$ & $a_{21}$ & $-\alpha_{2}$ & $a_{23}$ & 0 & $-a_{21}$ & 0 & 0 & $-a_{31}$ & 0 \\
$X_{22}$ & 0 & $-a_{12}$ & 0 & $a_{21}$ & 0 & $a_{23}$ & 0 & $-a_{32}$ & 0 \\
$X_{23}$ & 0 & $-a_{13}$ & 0 & 0 & $-a_{23}$ & 0 & $a_{21}$ & $\alpha_{3}-\alpha_{2}$ & $a_{23}$ \\
$X_{31}$ & $a_{31}$ & $a_{32}$ & $-\alpha_{3}$ & 0 & 0 & $-a_{21}$ & 0 & 0 & $-a_{31}$ \\
$X_{32}$ & 0 & 0 & $-a_{12}$ & $a_{31}$ & $a_{32}$ & $\alpha_{2}-\alpha_{3}$ & 0 & 0 & $-a_{32}$ \\
$X_{33}$ & 0 & 0 & $-a_{13}$ & 0 & 0 & $-a_{23}$ & $a_{31}$ & $a_{32}$ & 0
\end{tabular}

By replacing $Y$ by $Z, a$ by $b$ and $\alpha$ by $\beta$ in the above table we get the table for degree $(1,0,1)$.

By replacing $X$ by $Y, Y$ by $Z, a$ by $d, \alpha$ by $\delta$ in the above table we get the table for degree $(0,1,1)$.

Degree 3

Degree $(2,1,0)$.

\begin{tabular}{r|rrrrrrrr} 
& $\alpha_{2}$ & $a_{12}$ & $a_{13}$ & $a_{21}$ & $\alpha_{3}$ & $a_{23}$ & $a_{31}$ & $a_{32}$ \\
\hline$X_{11}$ & $\Upsilon_{X}$ & 0 & 0 & 0 & $\Upsilon_{X}$ & 0 & 0 & 0 \\
$X_{12}$ & 0 & 0 & 0 & $\Upsilon_{X}$ & 0 & 0 & 0 & 0 \\
$X_{13}$ & 0 & 0 & 0 & 0 & 0 & 0 & $\Upsilon_{X}$ & 0 \\
$X_{21}$ & 0 & $\Upsilon_{X}$ & 0 & 0 & 0 & 0 & 0 & 0 \\
$X_{22}$ & $-\Upsilon_{X}$ & 0 & 0 & 0 & 0 & 0 & 0 & 0 \\
$X_{23}$ & 0 & 0 & 0 & 0 & 0 & 0 & 0 & $\Upsilon_{X}$ \\
$X_{31}$ & 0 & 0 & $\Upsilon_{X}$ & 0 & 0 & 0 & 0 & 0 \\
$X_{32}$ & 0 & 0 & 0 & 0 & 0 & $\Upsilon_{X}$ & 0 & 0 \\
$X_{33}$ & 0 & 0 & 0 & 0 & $-\Upsilon_{X}$ & 0 & 0 & 0
\end{tabular}

Degrees $(1,2,0),(2,0,1),(1,0,2),(0,2,1)$ and $(0,1,2)$ are similar. 
Degree $(1,1,1)$.

\begin{tabular}{l|rrrrrrrr} 
& $\alpha_{2}$ & $a_{12}$ & $a_{13}$ & $a_{21}$ & $\alpha_{3}$ & $a_{23}$ & $a_{31}$ & $a_{32}$ \\
\hline$Z_{11}$ & $H$ & $\Gamma_{12}$ & $\Gamma_{13}$ & $-\Gamma_{21}$ & $H$ & 0 & $-\Gamma_{31}$ & 0 \\
$Z_{12}$ & $-2 \Gamma_{12}$ & 0 & 0 & $H_{12}$ & $-\Gamma_{12}$ & $\Gamma_{13}$ & $-\Gamma_{32}$ & 0 \\
$Z_{13}$ & $-\Gamma_{13}$ & 0 & 0 & $-\Gamma_{23}$ & $-2 \Gamma_{13}$ & 0 & $H_{13}$ & $\Gamma_{12}$ \\
$Z_{21}$ & $2 \Gamma_{21}$ & $H_{21}$ & $\Gamma_{23}$ & 0 & $\Gamma_{21}$ & 0 & 0 & $-\Gamma_{31}$ \\
$Z_{22}$ & $-H$ & $-\Gamma_{12}$ & 0 & $\Gamma_{21}$ & 0 & $\Gamma_{23}$ & 0 & $-\Gamma_{32}$ \\
$Z_{23}$ & $\Gamma_{23}$ & $-\Gamma_{13}$ & 0 & 0 & $-\Gamma_{23}$ & 0 & $\Gamma_{21}$ & $H_{23}$ \\
$Z_{31}$ & $\Gamma_{31}$ & $\Gamma_{32}$ & $H_{31}$ & 0 & $2 \Gamma_{31}$ & $-\Gamma_{21}$ & 0 & 0 \\
$Z_{32}$ & $-\Gamma_{32}$ & 0 & $-\Gamma_{12}$ & $\Gamma_{31}$ & $\Gamma_{32}$ & $H_{32}$ & 0 & 0 \\
$Z_{33}$ & 0 & 0 & $-\Gamma_{13}$ & 0 & $-H$ & $-\Gamma_{23}$ & $\Gamma_{31}$ & $\Gamma_{32}$
\end{tabular}

Where

$$
\begin{aligned}
& H_{21}=2 H-H_{12} \\
& H_{31}=2 H-H_{13} \\
& H_{32}=H-H_{13}+H_{12} \\
& H_{23}=H-H_{12}+H_{13} .
\end{aligned}
$$

\begin{tabular}{l|rrrrrrrr} 
& $\delta_{2}$ & $d_{12}$ & $d_{13}$ & $d_{21}$ & $\delta_{3}$ & $d_{23}$ & $d_{31}$ & $d_{32}$ \\
\hline$X_{11}$ & $F$ & $\Gamma_{12}$ & $\Gamma_{13}$ & $-\Gamma_{21}$ & $F$ & 0 & $-\Gamma_{31}$ & 0 \\
$X_{12}$ & $-2 \Gamma_{12}$ & 0 & 0 & $F_{12}$ & $-\Gamma_{12}$ & $\Gamma_{13}$ & $-\Gamma_{32}$ & 0 \\
$X_{13}$ & $-\Gamma_{13}$ & 0 & 0 & $-\Gamma_{23}$ & $-2 \Gamma_{13}$ & 0 & $F_{13}$ & $\Gamma_{12}$ \\
$X_{21}$ & $2 \Gamma_{21}$ & $F_{21}$ & $\Gamma_{23}$ & 0 & $\Gamma_{21}$ & 0 & 0 & $-\Gamma_{31}$ \\
$X_{22}$ & $-F$ & $-\Gamma_{12}$ & 0 & $\Gamma_{21}$ & 0 & $\Gamma_{23}$ & 0 & $-\Gamma_{32}$ \\
$X_{23}$ & $\Gamma_{23}$ & $-\Gamma_{13}$ & 0 & 0 & $-\Gamma_{23}$ & 0 & $\Gamma_{21}$ & $F_{23}$ \\
$X_{31}$ & $\Gamma_{31}$ & $\Gamma_{32}$ & $F_{31}$ & 0 & $2 \Gamma_{31}$ & $-\Gamma_{21}$ & 0 & 0 \\
$X_{32}$ & $-\Gamma_{32}$ & 0 & $-\Gamma_{12}$ & $\Gamma_{31}$ & $\Gamma_{32}$ & $F_{32}$ & 0 & 0 \\
$X_{33}$ & 0 & 0 & $-\Gamma_{13}$ & 0 & $-F$ & $-\Gamma_{23}$ & $\Gamma_{31}$ & $\Gamma_{32}$
\end{tabular}

Where

$$
\begin{aligned}
& F_{12}=F+H_{12}-H \\
& F_{21}=F+H-H_{12} \\
& F_{13}=F+H_{13}-H \\
& F_{31}=F+H-H_{13} \\
& F_{23}=F+H_{13}-H_{12} \\
& F_{32}=F+H_{12}-H_{13} .
\end{aligned}
$$




\begin{tabular}{l|rrrrrrrr} 
& $\beta_{2}$ & $b_{12}$ & $b_{13}$ & $b_{21}$ & $\beta_{3}$ & $b_{23}$ & $b_{31}$ & $b_{32}$ \\
\hline$Y_{11}$ & $F+H$ & $-\Gamma_{12}$ & $-\Gamma_{13}$ & $\Gamma_{21}$ & $F+H$ & 0 & $\Gamma_{31}$ & 0 \\
$Y_{12}$ & $2 \Gamma_{12}$ & 0 & 0 & $E_{12}$ & $\Gamma_{12}$ & $-\Gamma_{13}$ & $\Gamma_{32}$ & 0 \\
$Y_{13}$ & $\Gamma_{13}$ & 0 & 0 & $\Gamma_{23}$ & $2 \Gamma_{13}$ & 0 & $E_{13}$ & $-\Gamma_{12}$ \\
$Y_{21}$ & $-2 \Gamma_{21}$ & $E_{21}$ & $-\Gamma_{23}$ & 0 & $-\Gamma_{21}$ & 0 & 0 & $\Gamma_{31}$ \\
$Y_{22}$ & $-F-H$ & $\Gamma_{12}$ & 0 & $-\Gamma_{21}$ & 0 & $-\Gamma_{23}$ & 0 & $\Gamma_{32}$ \\
$Y_{23}$ & $-\Gamma_{23}$ & $\Gamma_{13}$ & 0 & 0 & $\Gamma_{23}$ & 0 & $-\Gamma_{21}$ & $E_{23}$ \\
$Y_{31}$ & $-\Gamma_{31}$ & $-\Gamma_{32}$ & $E_{31}$ & 0 & $-2 \Gamma_{31}$ & $\Gamma_{21}$ & 0 & 0 \\
$Y_{32}$ & $\Gamma_{32}$ & 0 & $\Gamma_{12}$ & $-\Gamma_{31}$ & $-\Gamma_{32}$ & $E_{32}$ & 0 & 0 \\
$Y_{33}$ & 0 & 0 & $\Gamma_{13}$ & 0 & $-F-H$ & $\Gamma_{23}$ & $-\Gamma_{31}$ & $-\Gamma_{32}$
\end{tabular}

Where

$$
\begin{aligned}
& E_{12}=F+2 H-H_{12} \\
& E_{21}=F+H_{12} \\
& E_{13}=F+2 H-H_{13} \\
& E_{31}=F+H_{13} \\
& E_{23}=F+H-H_{13}+H_{12} \\
& E_{32}=F+H-H_{12}+H_{13} .
\end{aligned}
$$

Degree 4

Degree $(1,1,2)$.

\begin{tabular}{l|rrrrrrrrrr} 
& $H_{12}$ & $\Gamma_{12}$ & $\Gamma_{13}$ & $\Gamma_{21}$ & $H_{13}$ & $\Gamma_{23}$ & $\Gamma_{31}$ & $\Gamma_{32}$ & $H$ & $F$ \\
\hline$Z_{11}$ & $\Phi_{Z}$ & 0 & 0 & 0 & $\Phi_{Z}$ & 0 & 0 & 0 & 0 & 0 \\
$Z_{12}$ & 0 & 0 & 0 & $\Phi_{Z}$ & 0 & 0 & 0 & 0 & 0 & 0 \\
$Z_{13}$ & 0 & 0 & 0 & 0 & 0 & 0 & $\Phi_{Z}$ & 0 & 0 & 0 \\
$Z_{21}$ & 0 & $\Phi_{Z}$ & 0 & 0 & 0 & 0 & 0 & 0 & 0 & 0 \\
$Z_{22}$ & $-\Phi_{Z}$ & 0 & 0 & 0 & 0 & 0 & 0 & 0 & 0 & 0 \\
$Z_{23}$ & 0 & 0 & 0 & 0 & 0 & 0 & 0 & $\Phi_{Z}$ & 0 & 0 \\
$Z_{31}$ & 0 & 0 & $\Phi_{Z}$ & 0 & 0 & 0 & 0 & 0 & 0 & 0 \\
$Z_{32}$ & 0 & 0 & 0 & 0 & 0 & $\Phi_{Z}$ & 0 & 0 & 0 & 0 \\
$Z_{33}$ & 0 & 0 & 0 & 0 & $-\Phi_{Z}$ & 0 & 0 & 0 & 0 & 0
\end{tabular}

Multiplication by the $X$ - and $Y$-variables is similar. Multiplying $\Upsilon_{X}, \Upsilon_{Y}$, $\Xi_{X}, \Xi_{Z}, \Lambda_{Y}, \Lambda_{Z}$ by the variables only results in zeros. 


\section{REFERENCES}

[1] F. Hreinsdóttir, The Koszul dual of the ring of commuting matrices, in Comm. Algebra 26 (1992), 3807-3819.

[2] C. Löfwall, J.E. Roos, A Nonnilpotent 1-2-presented Graded Hopf Algebra whose Hilbert Series Converges in the Unit Circle, Adv. in Math. 130 (2), 1997.

[3] C. Löfwall, On the subalgebra generated by one-dimensional elements in the Yoneda Ext-algebra, in: J.-E. Roos, ed., Algebra, Algebraic Topology and their Interactions, Lecture Notes in Math. 1183 (1986), 291-338.

DEPARTMENT OF MATHEMATICS

STOCKHOLM UNIVERSITY

S-10691 STOCKHOLM

SWEDEN

Email: freyja@matematik.su.se 